\title{
Activation of the Intrinsic Pain Inhibitory Circuit from the Midcingulate Cg2 to Zona Incerta Alleviates Neuropathic Pain
}

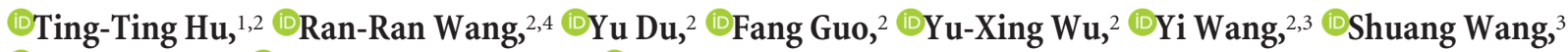 \\ ๑Xiang-Yao Li, ${ }^{2}$ @Shi-Hong Zhang, ${ }^{2}$ and $\mathbb{C}^{-Z h o n g}$ Chen, ${ }^{1,2,3}$ \\ ${ }^{1}$ Institute of Pharmacology and Toxicology, College of Pharmaceutical Sciences, Zhejiang University, Hangzhou 310058, China, ${ }^{2} \mathrm{NHC}$ and CAMS Key \\ Laboratory of Medical Neurobiology, School of Medicine, Zhejiang University, Hangzhou 310058, China, ${ }^{3}$ Department of Neurology, Second Affiliated \\ Hospital, School of Medicine, Zhejiang University, Hangzhou 310009, China, and ${ }^{4}$ Department of Pharmacy, Xinhua Hospital Affiliated to Shanghai Jiao \\ Tong University, School of Medicine, Shanghai 200092, China
}

Neuropathic pain is one of the most common and notorious neurological diseases. The changes in cerebral structures after nerve injury and the corresponding contributions to neuropathic pain are not well understood. Here we found that the majority of glutamatergic neurons in the area 2 of midcingulate cortex $\left(\mathrm{MCC} \mathrm{Cg2}{ }^{\text {Glu }}\right.$ ) were inhibited by painful stimulation in male mice. Optogenetic manipulation revealed that these neurons were tonically involved in the inhibitory modulation of multimodal nociception. We further identified the projections to GABAergic neurons in the zona incerta $\left(\mathrm{ZI}^{\mathrm{GABA}}\right)$ mediated the pain inhibitory role. However, $\mathrm{MCC} \mathrm{Cg} 2{ }^{\mathrm{Glu}}$ became hypoactive after nerve injury. Although a brief activation of the $\mathrm{MCC} \mathrm{Cg} 2{ }^{\mathrm{Glu}}$ to $\mathrm{ZI}{ }^{\mathrm{GABA}}$ circuit was able to relieve the aversiveness associated with spontaneous ongoing pain, consecutive activation of the circuit was required to alleviate neuropathic allodynia. In contrast, glutamatergic neurons in the area 1 of MCC played opposite roles in pain modulation. They became hyperactive after nerve injury and only consecutive inhibition of their activity relieved allodynia. These results demonstrate that $\mathrm{MCC} \mathrm{Cg} 2{ }^{\mathrm{Glu}}$ constitute a component of intrinsic pain inhibitory circuitry and their hypoactivity underlies neuropathic pain. We propose that selective and persistent activation of the $\mathrm{MCC} \mathrm{Cg} 2{ }^{\mathrm{Glu}}$ to $\mathrm{ZI}{ }^{\mathrm{GABA}}$ circuit may serve as a potential therapeutic strategy for this disease.

Key words: glutamatergic neurons; MCC Cg2; neuropathic pain; pain inhibitory circuitry; zona incerta

\section{Significance Statement}

Glutamatergic neurons in the area 2 of midcingulate cortex $\left(\mathrm{MCC} \mathrm{Cg}_{2}{ }^{\mathrm{Glu}}\right)$ are tonically involved in the intrinsic pain inhibition via projecting to GABAergic neurons in the zona incerta. They are hypoactive after nerve injury. Selective activation of the circuit compensates the reduction of its analgesic strength and relieves neuropathic pain. Therefore, $\mathrm{MCC} \mathrm{Cg2}{ }^{\mathrm{Glu}}$ and the related analgesic circuit may serve as therapeutic targets for neuropathic pain. In contrast, $\mathrm{MCC} \mathrm{Cg1}{ }^{\text {Glu }}$ have an opposite role in pain modulation and become hyperactive after nerve injury. The present study provides novel evidence for the concept that neuropathic pain is associated with the dysfunction of endogenous pain modulatory system and new perspective on the treatment of neuropathic pain.

\section{Introduction}

Neuropathic pain (NeuP) is a condition due to diseases or lesions affecting the somatosensory pathways. Despite the significant

Received July 18, 2019; revised Sept. 3, 2019; accepted Sept. 22, 2019.

Author contributions: T.-T.H., S.-H.Z., and Z.C. designed research; T.-T.H., R.-R.W., Y.D., F.G., Y.-X.W., Y.W., and

S.-H.Z. performed research; T.-T.H. and S.-H.Z. analyzed data; T.-T.H., S.-H.Z., Z.C., S.W., and X.-Y.L. wrote the paper.

This work was supported by Grants from National Natural Science Foundation of China $(81673404,81521062$,

81872843 ) and partly by 111 project (B13026).

The authors declare no competing financial interests.

Correspondence should be addressed to Zhong Chen at chenzhong@zju.edu.cn or Shi-Hong Zhang at shzhang713@zju.edu.cn.

https://doi.org/10.1523/JNEUROSCl.1683-19.2019

Copyright $\odot 2019$ the authors impact on life quality and the lack of effective treatment, the underlying mechanisms are still not fully understood. Several lines of evidence support that NeuP is accompanied with altered activity and connectivity of brain regions engaged in pain processing and modulation (Porreca et al., 2002; Mills et al., 2018; Patel et al., 2018; Yuan et al., 2018), but the relationship between these changes and failed pain control remains elusive.

Midcingulate cortex (MCC) participates in many brain functions, such as attention, cognition, affect and sensory processing, and its dysfunction has been implicated in several neurological or psychological disorders (Vogt, 2016b). Accumulating human brain imaging studies have identified MCC as a generalizable representation of acute nociceptive pain (Vogt, 2016a; Kragel et 
al., 2018). Intriguingly, changes in its activity (Kulkarni et al., 2007; Glass et al., 2011), connectivity (Fallon et al., 2016), and gray matter volume (Blankstein et al., 2010; Erpelding et al., 2016) under conditions of several types of chronic pain have been reported, indicating its vulnerability to chronic pain. However, MCC had previously been considered as the caudal part of anterior cingulate cortex (ACC) in rodents. Until in recent years has it been treated as a unique cingulate region in its own right. This change is largely attributed to a series of work of Vogt and his collogues, demonstrating that MCC has specific cytoarchitecture, neurochemistry and connectivity that are different from ACC (Vogt, 2016b). As a result, although numerous studies have focused on ACC (Bliss et al., 2016; Chen et al., 2018), evidence for the role of MCC in pain modulation and NeuP are still lacking.

A recent study using optogenetic approach without distinguishing subdivisions reported that MCC glutamatergic neurons do not mediate acute pain perception and associated affect, but gate capsaicin-induced sensory hypersensitivity, and that acutely silencing these neurons does not affect neuropathic allodynia (Tan et al., 2017). However, MCC comprises two subdivisions, area $1(\mathrm{Cg} 1)$ and area $2(\mathrm{Cg} 2)$, both of which have distinct afferents and efferents as well as neuronal density and topography (Vogt, 2016b; Fillinger et al., 2017, 2018), implying a functional heterogeneity within the MCC. Indeed, Hubbard et al. (2014) found that localized atrophy of ventral, rather than dorsal, anterior MCC correlates with catastrophizing of migraine, raising the possibility that MCC subdivisions may be differentially connected with pain. Here we report that glutamatergic neurons in MCC Cg2 (MCC Cg2 ${ }^{\mathrm{Glu}}$ ) constitute a component of the intrinsic pain inhibitory circuitry through projecting to GABAergic neurons in the zona incerta ( $\left.\mathrm{ZI}^{\mathrm{GABA}}\right)$. MCC $\mathrm{Cg} 2{ }^{\text {Glu }}$ become hypoactive in NeuP and optical activation of them alleviates mechanical allodynia and aversiveness associated with ongoing pain. In contrast, glutamatergic neurons in MCC Cg1 (MCC Cg1 ${ }^{\text {Glu }}$ ) have opposite roles in pain modulation and neuropathic pain. These results suggest that selective activation of the MCC $\mathrm{Cg} 2{ }^{\mathrm{Glu}}$ to ZI ${ }^{\text {GABA }}$ circuit may serve as a potential strategy for the treatment of NeuP.

\section{Materials and Methods}

Animals. Adult male mice were used in this study. C57BL/6J mice (http:// www.slaccas.com/product/details.asp?ProdId =2) were purchased from SLAC Laboratory Animal (http://www.slaccas.com/product/details.asp? ProdId=2). Vgat-ChR2-eYFP line 8 (https://www.jax.org/strain/014548) and Vgat-Cre (https://www.jax.org/strain/016962) breeders were purchased from The Jackson Laboratory and multiplied in the Experimental Animal Center, Zhejiang University, China. Animals were housed in a room with controlled humidity $(55 \pm 10 \%)$, room temperature $\left(22-24^{\circ} \mathrm{C}\right)$, and $12 \mathrm{~h}$ light/dark cycle (light on from 08:00 to 20:00). Food and water were available ad libitum. All procedures were in accordance with the guidelines of the International Association for the Study of Pain (Zimmermann, 1983) and were approved by the Zhejiang University Animal Experimentation Committee. Efforts were made to ensure minimal animal use and discomfort.

Surgical procedures. Stereotaxic surgery was done on mice under sodium pentobarbital anesthesia (50 mg/kg, i.p.). The mouse was mounted in a stereotaxic apparatus (Stoelting) and the body temperature was maintained by a heating pad. Small craniotomies were made over the target areas either for immediate viral delivery or implantation of guide cannula [outer diameter (o.d.): $0.41 \mathrm{~mm}$; RWD] or chronic fiber optic cannula (core diameter: $200 \mu \mathrm{m}, 0.22 \mathrm{NA}$; Newdoon). The coordinates relative to bregma were as follows according to the Paxinos and Franklin (2001) atlas : left MCC Cg2 (AP: $0.2 \mathrm{~mm}$, ML: $0.3 \mathrm{~mm}$, DV: $1.9 \mathrm{~mm}$ ), left MCC Cg1 (AP: $0.2 \mathrm{~mm}$, ML: $0.3 \mathrm{~mm}$, DV: $1.2 \mathrm{~mm}$ ), left ZI (AP: -1.7 $\mathrm{mm}, \mathrm{ML}: 1.2 \mathrm{~mm}, \mathrm{DV}: 4.4 \mathrm{~mm}$ ), and left dorsal lateral periaqueductal gray (DLPAG; AP: $-3.7 \mathrm{~mm}$, ML: $0.4 \mathrm{~mm}$, DV: $2.4 \mathrm{~mm}$ ). The cannulas were held in place by dental acrylic and the patency was maintained with occlusion stylets. The sites of cannula placement or viral expression were histochemically confirmed on cryogenic brain sections at the end of all experiments. The core of viral expression should be within the target nuclei according to the brain atlas and be matched with the cannula tips. Animals displaying incorrect sites were excluded from all analysis.

Partial sciatic ligation model (PSL) of neuropathic pain was induced as described previously (Seltzer et al., 1990). In brief, mice were anesthetized by isoflurane inhalation ( $4 \%$ for induction and $2 \%$ for maintenance) and were placed on a heating pad to maintain the body temperature. Under aseptic condition and a surgical microscope, the right sciatic nerve trunk was exposed. An 8-0 silk suture was inserted into the dorsum of the nerve with a $3 / 8$ curved, reversed-cutting mini-needle and was tightly ligated so that the dorsal $1 / 3-1 / 2$ of the nerve thickness was trapped in the ligature. The nerve in the sham group was exposed but left intact. The wound was closed in layers. Animals were placed back to their individual cages after recovered from anesthesia in a warm incubator (Harvard Apparatus)

Delivery of viruses and agents. After the small craniotomies performed as described, a thin glass capillary connected to a $1 \mu \mathrm{l}$ microsyringe that was mounted on an Ultra Micro Pump (WPI) was slowly lowered to the target sites. Virus at a total volume of $0.1 \mu \mathrm{l}$ was delivered at a flow rate of $10 \mathrm{nl} / \mathrm{min}$. The glass capillary was left in place for an additional $5 \mathrm{~min}$ after injection to allow the diffusion of virus particles and minimize the reflux along the injection track. For optical activation or inhibition of glutamatergic neurons, pAAV-CaMKII $\alpha$-hChR2(H134R)-eYFP (ChR2 virus in brief thereafter, viral titers: $1.7 \times 10^{13}$ particles/ml; OBIO) or pAAV-CaMKII $\alpha$-eArch3.0-eYFP (Arch virus in brief thereafter, viral titers: $1.3 \times 10^{12}$ particles $/ \mathrm{ml}$; OBIO) was infused to the target areas of $\mathrm{C} 57 \mathrm{BL} / 6 \mathrm{~J}$ mice. The specific transduction of viruses in glutamatergic neurons was confirmed by the colocalization of eYFP and CaMKII $\alpha$. For optical inhibition of GABAergic neurons in the ZI, AAV-CAG-FLEXArchT-GFP (viral titers: $2.14 \times 10^{13} \mathrm{vg} / \mathrm{ml}$; OBIO) was injected to the ZI of Vgat-Cre mice. Animals were kept for 4 weeks to allow the maximal in vivo viral expression before subsequent experiments. For chemogenetic inhibition of GABAergic neurons in the ZI, AAV-EF1a-DIO-hM4DimCherry (viral titers: $1.4 \times 10^{13} \mathrm{vg} / \mathrm{ml}$; OBIO) whose expression was controlled in a Cre-dependent manner was injected to the ZI of Vgat-Cre mice. Four weeks later, all mice received clozapine- $N$-oxide $(\mathrm{CNO} ; 1$ $\mathrm{mg} / \mathrm{kg}$, i.p.; Abcam) injection $30 \mathrm{~min}$ before the behavioral test.

For retrograde trans-synaptic tracing, $0.2 \mu \mathrm{l}$ viral mixture of rAAVEF1a-DIO-His-EGFP-2 $\alpha$-TVA-WPRE-pA (viral titers: $3.77 \times 10^{12} \mathrm{vg} /$ $\mathrm{ml}$; BrainVTA) and rAAV-EF1a-DIO-RVG-WPRE-pA (viral titers: $3.43 \times 10^{12} \mathrm{vg} / \mathrm{ml}$; BrainVTA) mixed at 1:1 were microinjected into the ZI of Vgat-Cre mice. Three weeks later, $0.2 \mu \mathrm{l}$ modified rabies virus RV-EnvA- $\triangle$ G-DsRed (viral titers: $7.50 \times 10^{8} \mathrm{vg} / \mathrm{ml}$; BrainVTA) was microinjected at the same site and mice were killed another week later.

For blocking local glutamatergic neurotransmission, $0.5 \mu \mathrm{l}$ mixed solution containing CNQX (0.5 $\mu \mathrm{g}$; Sigma-Aldrich), a glutamate AMPA receptor antagonist, and AP5 (4 $\mu \mathrm{g}$; Abcam), a NMDA receptor antagonist, was infused into the MCC Cg2 or ZI slowly over a period of $2 \mathrm{~min}$ via a 33 gauge needle that fits the guide cannula. Behavioral tests were performed 15 min later.

In vivo optical stimulation. The chronic fiber optic cannula was connected to a portable optogenetic system through an optogenetic patch cord (Newdoon). To examine the instant effect of optical stimulation, persistent laser stimulation at $473 \mathrm{~nm}$ (blue: power $5 \mathrm{~mW}$, frequency 10 $\mathrm{Hz}$, pulse width $10 \mathrm{~ms}$ ) or $594 \mathrm{~nm}$ (yellow: $5 \mathrm{~mW}$, direct current) was supplied to activate or inhibit neurons, respectively, throughout the behavioral testing. For chronic optical stimulation through postoperative Days 1-14, the laser was delivered daily at a $30 \mathrm{~s}$ on/off cycle lasting $900 \mathrm{~s}$. Such a cyclic stimulation pattern was adopted to minimize the possible brain tissue damage caused by long-lasting illumination. The frequency of $10 \mathrm{~Hz}$ for blue laser was adopted based on the findings in a pilot study that $20 \mathrm{~Hz}$ stimulation of MCC Cg2 ${ }^{\text {Glu }}$ increased mechanical thresholds in bilateral hindpaws similarly to $10 \mathrm{~Hz}$, but evoked seizures in some animals. A preliminary experiment found that neither ChR2 virus infusion into the MCC alone nor illumination of MCC with ChR2 virus 
infusion by yellow laser affected mechanical sensitivity. Thus, mice receiving viruses that were connected with the optic fiber but without illumination served as the control for those receiving both viruses and illumination.

Behavioral tests. All behavioral experiments were performed by female experimenters blinded to treatment conditions. Pain-like behaviors were tested in intact mice or mice receiving PSL or sham operation on Days 7 and 14 postoperatively.

For evaluation of mechanical sensitivity, we adopted the simplified up-down method for estimating paw withdrawal threshold (PWT) with a set of von Frey filaments (Bonin et al., 2014). In detail, mice were acclimated for $1 \mathrm{~h}$ in an elevated cage with a wire mesh floor. A series of von Frey filaments numbered 2 through 9 with bending force from 0.02 to $1.4 \mathrm{~g}$ were applied to the plantar surface of the hindpaw for $3 \mathrm{~s}$. Testing began with filament number 5 and progressed according to an up-down method. Each test constituted a constant number of five stimuli. A sharp withdrawal or an immediate flinch of the hindpaw indicated a positive response. PWT estimate was calculated by adding an adjustment value of \pm 0.5 to the value of the fifth filament used in each test. The adjustment factor was positive if there was no response to the fifth filament of the sequence to generate a PWT slightly higher than the fifth filament value, or negative if there was a withdrawal to generate a PWT slightly lower than the fifth filament value. PWT was converted from filament number to force, and expressed in grams using the equation: $\mathrm{PWT}_{\text {force }}=$ $10^{(x \times F+B)}$, where $x$ was 0.24 and $B$ was -2.00 . In some cases, the mechanical sensitivity was evaluated by measuring the paw withdrawal latency (PWL) to the stimulation of the filament with $0.07 \mathrm{~g}$ bending force.

Hargreaves test and hotplate test were used for the evaluation of thermal nociception. For the former, mice were placed in a transparent Plexiglas chamber with a glass bottom and were allowed to acclimatize for $1 \mathrm{~h}$ before the test. A radiant heat source (Ugo Basile) with an intensity of 20 $\mathrm{W}$ was positioned underneath the glass bottom and aimed at the plantar surface of the hindpaw. PWL was recorded automatically. Minimum and maximum cutoffs were assigned at 1 and $20 \mathrm{~s}$, respectively. The trial was repeated at a $15 \mathrm{~min}$ interval on each paw, and the average of two trials was taken into analysis. For the hotplate test, mice were placed on the hotplate surface with the temperature set at $50.0 \pm 0.1^{\circ} \mathrm{C}$. Movement of the mouse on the plate was constrained by a Plexiglas cylinder (diameter $20 \mathrm{~cm}$ ). The latency to a withdrawal response like licking, flicking or lifting the hindpaw was recorded and the cutoff time was set at $30 \mathrm{~s}$. Each test consisted of two separate trials with $15 \mathrm{~min}$ interval and the latencies were averaged as PWL.

For formalin test, $20 \mu \mathrm{l}$ of $2 \%$ formalin was administered subcutaneously into the dorsal surface of the hindpaw after mice were acclimatized in a glass cage $(28 \times 17 \times 13 \mathrm{~cm})$ for $20 \mathrm{~min}$. The duration of paw biting, licking, and shaking in Phase 1 (0-5 min) and Phase 2 (15-50 min) was timed with a handheld stopwatch.

The conditioned place preference (CPP) test was used to evaluate the affective responses to optogenetic manipulation. According to King et al. (2009), if a certain treatment alleviates spontaneous ongoing pain, mice will show preference to the place paired with the treatment. This experiment is based on the hypothesis that relief of pain is rewarding. On postoperative Day 14, mice receiving PSL surgery or sham operation were preconditioned in an automated three-chamber CPP box across $2 \mathrm{~d}$ and were allowed to explore the box freely for $30 \mathrm{~min}$ each day. Animal behaviors on the second day were recorded by a digital video camera (Logitech) and the data in the first 15 min were analyzed by the ANYmaze software to verify the absence of preference to any chamber. Animals spending $>80 \%(>720$ s) or $<20 \%(<180$ s) of the total time in any of the chambers were eliminated from further testing. On Day 3, mice were placed in a chamber on a randomly chosen side paired with laser off treatment in the morning, and were placed in the chamber on the other side paired with laser on treatment $4 \mathrm{~h}$ later in the afternoon. On Day 4 , mice were placed in the chamber paired with laser on in the morning, and were placed in the one paired with laser off $4 \mathrm{~h}$ later in the afternoon. Mice were not allowed to access the other chambers after being placed in either chamber. On Day 5, animals were placed in the middle chamber with free access to chambers on both sides. The time they spent in each chamber in a period of 15 min was recorded.
Fiber photometry. The virus pAOV-CaMKII $\alpha$-GCAMP6(s)-eYFP was infused into the left MCC Cg2 as described in the section of Delivery of viruses and agents. Three weeks later, an optic fiber $(0.20 \mathrm{~mm}$ o.d., NA $=$ 0.37 ; Inper) placed in a ceramic ferrule was inserted into the MCC Cg2. The mice were housed individually for at least 1 week for recovery. The fiber photometry recording system (Thinkertech) used a dichroic mirror (MD498, Thorlabs) to reflect a $488 \mathrm{~nm}$ laser (OBIS 488LS, Coherent), which was focused with a $10 \times$ objective lens $(\mathrm{NA}=0.3$; Olympus $)$ and then coupled to an optical commutator (Doric Lenses). The commutator and the implanted fiber were guided by a fiber cable $(200 \mathrm{~mm}$ o.d., NA $=$ 0.37). GCaMP fluorescence was filtered using a GFP bandpass filter and collected using a photomultiplier tube (R3896, Hamamatsu). The amplifier converted the PMT current output into a voltage signal that was further filtered by a low-pass filter (4 Hz cutoff; Brownlee, 440). The analog voltage signals were digitized at $500 \mathrm{~Hz}$ and recorded. The mice were connected to the fiber photometry setup after acclimatized in a glass cage $(28 \times 17 \times 13 \mathrm{~cm})$ for $20 \mathrm{~min}$ and the baseline signals were recorded for $5 \mathrm{~min}$ before the subcutaneous injection of $20 \mu \mathrm{l}$ of $2 \%$ formalin or saline into the dorsal surface of the right hindpaw. The animals were returned to their original cages and the signals were recorded continuously for another $50 \mathrm{~min}$. Photometry data were exported as MATLAB files for further analysis. We derived the values of fluorescence change $(\Delta F / F)$ by calculating $\left(F-F_{0}\right) / F_{0}$, where $F_{0}$ is the averaged fluorescence over the 5 min baseline period. $\Delta F / F$ values for each mouse were presented as heatmaps and the averaged values were presented in plots with the SEM indicated by a shaded area.

In vivo single-unit recordings. Mice were anesthetized with $20 \%$ urethane $(1.4 \mathrm{~g} / \mathrm{kg}$, i.p.; Sigma-Aldrich) and mounted on a stereotactic frame. A small craniotomy was performed over the left MCC or ZI. A bundle of microelectrodes consisting of 8 channels of wires $(25 \mu \mathrm{m}$; AM-Systems) with impedances of $1-2 \mathrm{M} \Omega$ was lowered to the target area by a micromanipulator. An optical fiber was bound in the microelectrode bundle in the case of recording the responses of neurons to illumination. Acquisition and analysis of data from single-unit recordings were conducted as previously described (Wang et al., 2017). In short, signals were acquired by a multichannel acquisition system (Blackrock Microsystems) with a sampling rate of $30 \mathrm{kHz}$ and were high- and low-passed at $250 \mathrm{~Hz}$ and $7.5 \mathrm{kHz}$, respectively. Units were selected if the signal-tonoise voltage threshold was $>3: 1$. Recordings were analyzed by Offline Sorter (Plexon) and NeuroExplorer 4.0 (NEX). Putative glutamatergic neurons were identified by their wide spike waveform (full-width at halfmaximum $\geq 0.30 \mathrm{~ms}$ ) and sharp autocorrelation (Csicsvari et al., 1999; Le Van Quyen et al., 2008). The spontaneous and evoked firing of left MCC Cg2 ${ }^{\text {Glu }}$ and Cg1 ${ }^{\text {Glu }}$ were recorded $14 \mathrm{~d}$ after sham or PSL operation. The spontaneous firing was recorded for $2 \mathrm{~min}$ before recording the evoked firing by noxious pinch stimulation. Pinch stimulation was applied by clipping the paw for $30 \mathrm{~s}$ using a toothed plastic clip. Formalin was administered into the dorsal surface of the right hindpaw after recording the baseline firing for $5 \mathrm{~min}$. ZI neurons were identified according to a matching depth reading and evoked responses by contralateral whisker stimulation, which ZI is known to possess (Nicolelis et al., 1992).

Histological examination. Mice were deeply anesthetized with pentobarbital (100 mg/kg, i.p.), and perfused intracardially with ice-cold PBS followed by $4 \%$ paraformaldehyde, $\mathrm{pH}$ 7.4. Brains were removed and postfixed overnight in the same fixative, then dehydrated by infiltration in $30 \%$ sucrose for $48 \mathrm{~h}$ at $4^{\circ} \mathrm{C}$. Coronal brain sections were cut at $30 \mu \mathrm{m}$ on a cryostat (ThermoFisher Scientific) and stored at $-80^{\circ} \mathrm{C}$. Toluidine blue staining was used for the examination of electrode/cannula placement sites. Fluorescent images were captured by a fluorescence microscope (Olympus). For immunohistochemical staining, sections were first incubated with $5 \%$ donkey serum albumin for $2 \mathrm{~h}$ after incubation with $0.1 \%$ Triton X-100 for $15 \mathrm{~min}$ at room temperature (RT), and then were incubated overnight at $4^{\circ} \mathrm{C}$ with rabbit anti-CaMKII $\alpha$ (1:200; Abcam, catalog \#ab134041; RRID:AB_2811181). After washing three times for 5 min with PBS, the sections were incubated with donkey anti-rabbit AlexaFluor 594- (Jackson ImmunoResearch, catalog \#706-586-148; RRID: AB_2340475) or AlexaFluor 488- (Jackson ImmunoResearch, catalog \#711-545-152; RRID:AB_2313584) conjugated secondary fluorescent 
antibody for $2 \mathrm{~h}$ at RT. After repeated washing, the sections were then covered with glass coverslips and images were captured by a laser confocal microscope (Leica).

Experimental design and statistical analysis. The experimental design was as follows. Data from 655 male mice (of 750 mice) were included in data analysis. All excluded animals displayed incorrect sites for cannula placement or viral expression. Four mice were excluded from hotplate test because the PWL was $>30 \mathrm{~s}$.

Changes in MCC Cg2 ${ }^{\text {Glu }}$ activity upon noxious stimulation under intact and neuropathic conditions (see Fig. 1). We first recorded the firing of MCC Cg2 ${ }^{\mathrm{Glu}}$ in mice receiving sham ( $n=31$ from 4 mice) and PSL ( $n=38$ from 5 mice) surgery before and after pinch stimulation of the hindpaw, taking $\mathrm{Cg} 1{ }^{\text {Glu }}$ as a comparison $(n=21$ from 3 sham mice and $n=20$ from 3 PSL mice). We further verified the nociceptionassociated changes in MCC Cg2 ${ }^{\text {Glu }}$ activity in the formalin test by measuring the calcium signals ( $n=4$ and 5 for saline and formalin groups, respectively) as well as the firing frequency ( $n=11$ from 9 mice) before and after formalin injection in the hindpaw.

Effects of selective activation and inhibition of MCC $\mathrm{Cg} 2{ }^{\mathrm{Glu}}$ on nociception (see Fig. 2). Mice receiving ChR2 virus targeting MCC Cg2 Glu were divided into two groups. One group was subjected to von Frey test, Hargreaves test and hotplate test in sequence with an interval of $2 \mathrm{~d}$ ( 20 of 26 mice, $n=10$ for both laser on and laser off in all tests, except for $n=$ 9 in hotplate test). The other group was used for the formalin test in the ipsilateral (17 of 22 mice, $n=8$ and 9 for laser on and laser off, respectively) and contralateral hindpaw ( 16 of 22 mice, $n=8$ for both laser on and laser off). Mice receiving Arch virus targeting MCC Cg2 ${ }^{\mathrm{Glu}}$ were subjected to mechanical and thermal nociception as those receiving ChR2 virus ( 20 of 28 mice, $n=10$ for laser on and laser off, respectively, in all tests, except for $n=8$ for laser off in hotplate test).

Identification of the circuit mediating the pain modulatory role of MCC Cg2 ${ }^{\text {Glu }}$ (see Fig. 3). We first tested the effect of local injection of saline or CNQX + AP5 in von Frey test ( 34 of 40 mice, $n=8$ for both laser on and off in saline group; $n=9$ for both laser on and off in CNQX + AP5 group) and formalin test ( 28 of 40 mice, $n=6$ and 8 for laser on and off in both saline and CNQX+AP5 groups). We then tested the respective role of MCC Cg2 ${ }^{\text {Glu }}$ terminals in the ZI ( 19 of 20 mice, $n=10$ and 9 for laser on and laser off, respectively) and DLPAG (19 of 20 mice, $n=9$ and 10 for laser on and laser off, respectively) in the analgesic effect and further tested whether the effect of MCC $\mathrm{Cg} 2{ }^{\mathrm{Glu}}$ terminals in the $\mathrm{ZI}$ is glutamate dependent in von Frey test (36 of 40 mice, $n=8$ and 10 for both laser on and off in saline and CNQX + AP5 groups) and formalin test ( 25 of 32 mice, $n=6$ and 7 for laser on and off in saline group, respectively; $n=6$ for both laser on and off in CNQX+AP5 group).

Association of MCC Cg2 ${ }^{\text {Glu }}$ with $\mathrm{ZI}^{\mathrm{GABA}}$ (see Fig. 4). We first tested the activation of $\mathrm{ZI}{ }^{\mathrm{GABA}}$ by MCC Cg $2{ }^{\mathrm{Glu}}$ ( $n=32$ from 4 mice), then did trans-synaptic tracing of presynaptic inputs to $\mathrm{ZI}^{\mathrm{GABA}}(n=4)$. We also tested the effect of selectively modulating $\mathrm{ZI}{ }^{\mathrm{GABA}}$ on mechanical nociception in intact Vgat-ChR2 mice ( 15 of 16 mice, $n=7$ and 8 for laser on and laser off, respectively) and Vgat-Cre mice (17 of 18 mice, $n=7$ and 10 for laser on and laser off, respectively), as well as the effect of selectively inhibiting $\mathrm{ZI}{ }^{\mathrm{GABA}}$ by chemogenetic approach and optically activating ZI terminals of MCC Cg2 ${ }^{\text {Glu }}$ simultaneously ( 28 of 35 mice, $n=7$ for both laser on and laser off without $\mathrm{CNO} ; n=6$ for laser on $+\mathrm{CNO} ; n=8$ for CNO alone).

Effect of modulating the MCC $\mathrm{Cg} 2{ }^{\mathrm{Glu}}$ to $\mathrm{ZI}{ }^{\mathrm{GABA}}$ circuit on neuropathic pain (see Fig. 5). The effects in sham- and PSL-treated mice were evaluated by von Frey test and CPP test. Laser stimulation was either given once on the test day or once daily throughout the postoperative days. We first modulated MCC Cg2 ${ }^{\text {Glu }}$ activity (in von Frey test: for photoactivation: 36 of 38 mice, $n=8$ for both sham and laser on daily groups, $n=10$ for both laser off and laser on once groups; for photoinhibition: 38 of 38 mice, $n=8$ for sham group, $n=10$ for laser off, laser on once and laser on daily groups, respectively; in CPP test: for photoactivation: 16 of 20 mice, $n=8$ for both sham and PSL groups; for photoinhibition: 13 of 16 mice, $n=7$ and 6 for two groups, respectively). We then selectively activated MCC Cg2 ${ }^{\text {Glu }}$ terminals in the ZI (in von Frey test: 31 of 34 mice, $n=8$ for sham group, $n=9$ for laser off group, $n=$ 7 for both laser on once and laser on daily groups; in CPP test: 13 of 16 mice, $n=6$ and 7 for sham and PSL groups, respectively) and in the DLPAG (in von Frey test: 34 of 34 mice, $n=8$ for sham, laser on once and laser on daily groups, respectively, $n=10$ for laser off group; in CPP test: 13 of 16 mice, $n=7$ and 6 for sham and PSL groups, respectively).

Effect of activating ZI ${ }^{\mathrm{GABA}}$ on neuropathic pain (see Fig. 6). The effect in sham- and PSL-treated Vgat-ChR2-eYFP mice was evaluated by von Frey test ( 25 of 26 mice, $n=10,8$ and 7 for sham, laser off and laser on groups, respectively) and CPP test ( 17 of 18 mice, $n=7$ and 10 for sham and PSL groups, respectively).

Effect of modulating MCC Cg1 ${ }^{\text {Glu }}$ on nociception and neuropathic pain (see Fig. 7). The effect in intact and PSL-treated mice was evaluated by von Frey test and CPP test. Laser stimulation was either given once on the test day or once daily throughout the postoperative days. We first selectively activated MCC Cg1 ${ }^{\text {Glu }}$ (in von Frey test: 28 of 30 mice, $n=10$ for both laser on and laser off in intact mice, $n=10$ for laser off group, $n=9$ for both laser on once and laser on daily groups in neuropathic mice; in CPP test: 13 of 16 mice, $n=7$ and 6 for sham and PSL groups, respectively), then selectively inhibited them (in von Frey test: 27 of 32 mice, $n=10$ for both laser on and laser off in intact mice, $n=10,9$ and 8 for laser off, laser on once and laser on daily groups, respectively, in neuropathic mice; in CPP test: 16 of 20 mice, $n=6$ and 10 for sham and PSL groups, respectively).

Neuronal firing was quantified using the average firing frequency during each specified time interval. The responses of neurons to stimulation were classified into three categories, i.e., activation, inhibition and no change. Activation or inhibition was defined if the firing frequency increased or reduced by at least $20 \%$ during the stimulation compared with the baseline, respectively, whereas no change was defined if the firing frequency changed by no $>20 \%$.

Data are presented as mean \pm SEM. Statistical analysis was conducted by Prism GraphPad v8.0 and SPSS v16.0 for Windows. Paired $t$ test was used to compare the baseline and evoked firing frequency of the same neuron. Unpaired $t$ test was used to compare the firing frequency and pain-like behavioral data of the sham and neuropathic groups, whereas one-way ANOVA with Tukey's post hoc was used when appropriate to compare among three groups. Two-way ANOVA with repeated measures and Bonferroni's post-test was used to test the interaction of laser stimulation and side and compare between groups for behavioral tests in bilateral hindpaws. Fisher's exact test was used to compare the proportions of different types of neurons in sham and neuropathic groups. For CPP test, differences in time spent in each chamber during preconditioning and after conditioning test were analyzed using two-way ANOVA with repeated measures and Bonferroni's post-test. All comparisons were two-tailed and the significance level was set at $p<0.05$.

\section{Results \\ MCC Cg2 ${ }^{\text {Glu }}$ are inhibited by noxious stimulation and neuropathic pain}

Imaging studies have reported that MCC neurons can be activated by exogenous stimuli (Vogt, 2005). Here we first examined specifically whether MCC glutamatergic neurons respond to painful stimulation in anesthetized mice from sham and PSL groups by in vivo single-unit recording on postoperative Day 14. The glutamatergic nature of recorded neurons was identified by the characteristic waveform and the autocorrelation of neuronal discharge (Fig. 1A). We found that MCC glutamatergic neurons responded to noxious pinch stimulation in three patterns: activation, inhibition and no change (Fig. $1 B, C$ ). In sham-treated mice, the majority of $\mathrm{Cg} 1{ }^{\text {Glu }}$ and $\mathrm{Cg} 2$ Glu $(76$ and $65 \%$, respectively) were inhibited, which was also manifested by the reduced average firing frequency (Fig. $1 D$; paired $t$ test, pinch vs pre, $p<$ 0.001 and $=0.0238$, respectively). In neuropathic mice, more $\mathrm{Cg} 1{ }^{\text {Glu }}$ were activated by pinch stimulation ( 35 vs $5 \%$, Fisher's exact test, PSL vs sham, $p=0.047$ ), whereas the proportion of three response patterns of $\mathrm{Cg} 2{ }^{\text {Glu }}$ remained unchanged, although there was a trend that more neurons were inhibited ( 82 vs $65 \%$; Fig. $1 B, C)$. Interestingly, the activity of MCC glutamatergic neu- 
A

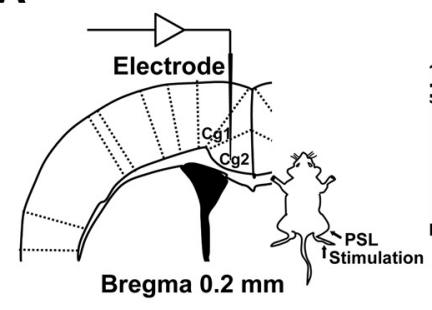

C

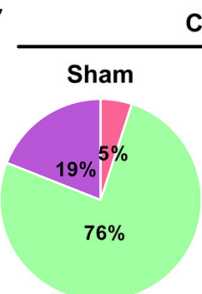

Total $n=21$ g1

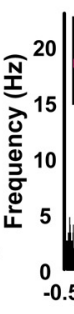

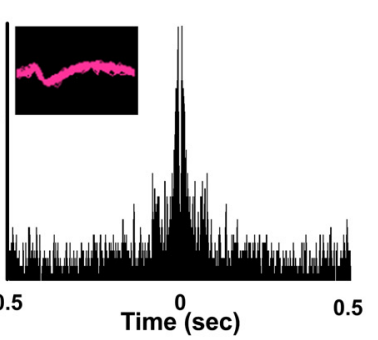

B

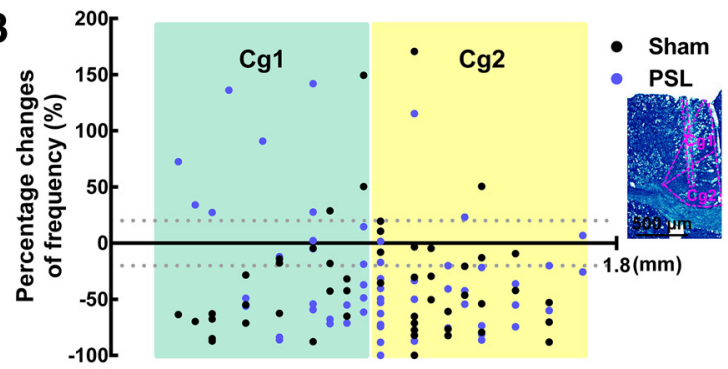

E
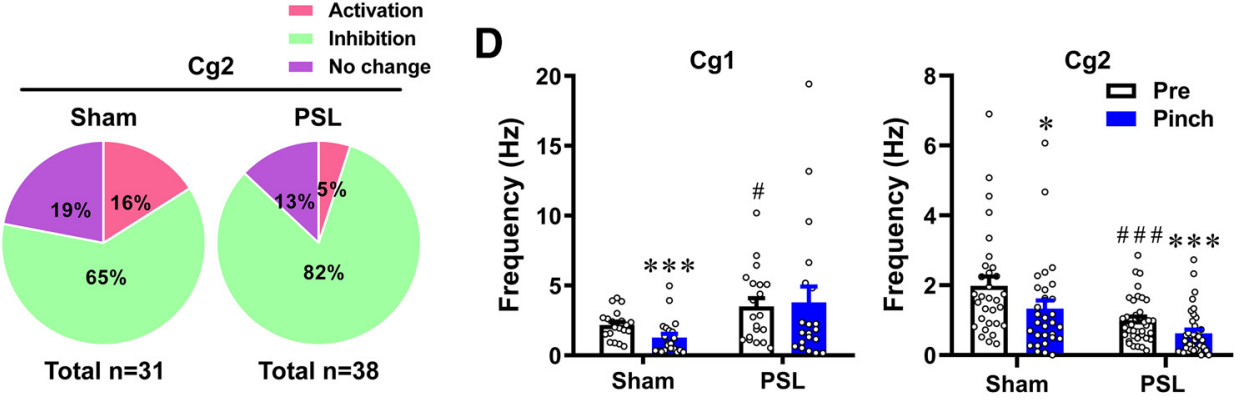

F
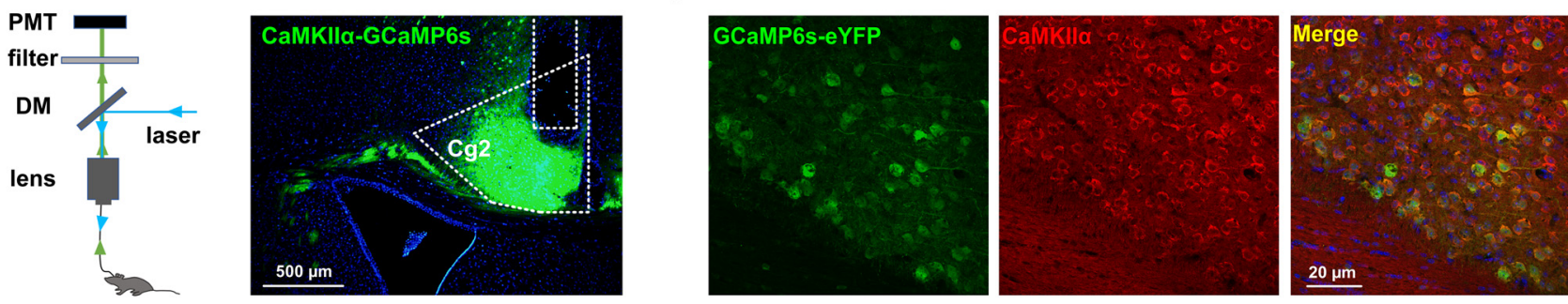

G

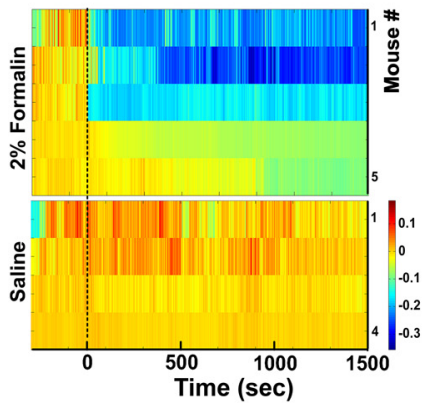

H

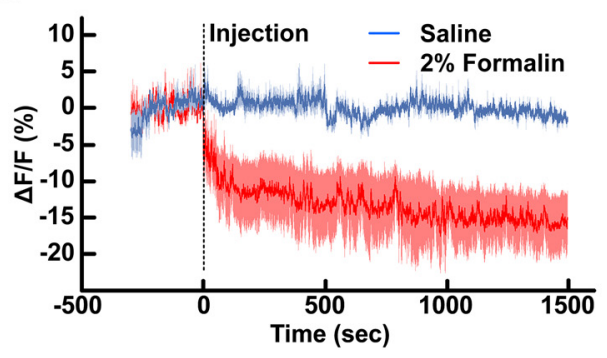

I

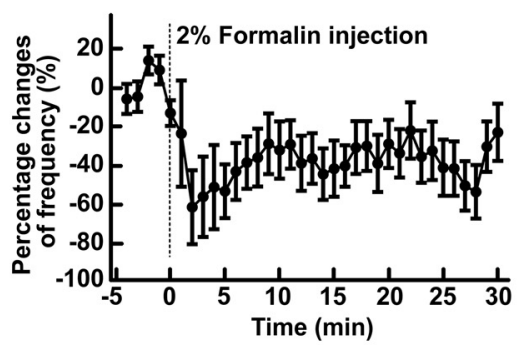

Figure 1. $M C C \mathrm{Cg}^{\text {Glu }}$ are inhibited by painful stimulation. $A$, Schematic drawing of the electrode placement in the left MCC, PSL surgery, and stimulation of the hindpaw on the right side (left), and the waveform and autocorrelation of a representative glutamatergic neuron (right). $\boldsymbol{B}$, Mapping of the percentage changes of firing frequency of all recorded MCC (AP: $0.2 \mathrm{~mm}, \mathrm{ML}: 0.3 \mathrm{~mm}$ ) glutamatergic neurons in response to pinch stimulation and their corresponding depth relative to the brain surface in sham- and PSL-treated mice (left), and representative image of the electrode trace in the MCC (right). Scale bar, $500 \mu \mathrm{m}$. C, Proportion of MCC glutamatergic neurons that increased, decreased, and remained their firing frequency upon pinch stimulation in sham- and PSL-treated mice. For C $\mathrm{g} 1$, Fisher's exact test, PSL versus sham, $p=0.047$. D, Changes in firing frequency of MCC glutamatergic neurons in response to pinch stimulation in sham- and PSL-treated mice. Two-tailed unpaired $t$ test for PSL versus sham, two-tailed paired $t$ test for pinch versus pre. For Cg1: $\operatorname{sham} n=21$, pinch versus pre, $p<0.001, t=5.316, \mathrm{df}=20$. PSL $n=20$, pinch versus pre, $p=0.7091, t=0.3787, \mathrm{df}=19$; PSL versus sham, $p=0.0335, t=2.204, \mathrm{df}=39$. For Cg2: sham $n=31$, pinch versus pre, $p=0.0238, t=2.380, \mathrm{df}=30$. PSL $n=38$, pinch versus pre, $p<0.001, t=4.901, \mathrm{df}=37$; PSL versus sham, $p<0.001, t=3.681, \mathrm{df}=67$. E, Schematic drawing of the fiber photometry setup (left) and photomicrograph showing the GCaMP6s-eYFP expression in MCC Cg2 (right). Scale bar, $500 \mu \mathrm{m}$. DM, Dichroic mirror; PMT, photomultiplier tube. $F$, Representative images showing the colocalization of GCaMP6s-eYFP in MCCCg2 with CaMKIl $\alpha$, a marker for glutamatergic neurons. Scale bar, $20 \mu \mathrm{m}$. G, Heatmaps of GCaMP6s fluorescence changes $(\Delta F / F)$ in MCC $C g{ }^{\text {Glu }}$ in each individual mouse receiving saline or formalin injection in the contralateral hindpaw. $\boldsymbol{H}$, Peri-event plot of GCaMP6s $\Delta F / F$ after saline or formalin injection ( $n=4$ and 5 , respectively). $\boldsymbol{I}$, Percentage change of firing frequency (time bin $=1$ min) of MCC $\mathrm{Cg} 2$ Glu after formalin injection. $n=11 . \mathbf{G}-I$, Dashed lines indicate injection. ${ }^{*} p<0.05$, ${ }^{* * *} p<0.001$, respectively, pinch versus pre; \#p $<0.05$ and \#\# \# $<0.001$, respectively, PSL versus sham.

rons changed under neuropathic condition. Compared with sham-treated mice, the frequency of spontaneous firing in neuropathic mice was higher in $\mathrm{Cg} 1 \mathrm{Glu}$, but was lower in Cg2 Glu (Fig. $1 D$; unpaired $t$ test, PSL vs sham, $p=0.0335$ and $<0.001$, respectively), suggesting these neurons become hyperactive and hypoactive, respectively. The decline in firing frequency evoked by pinch stimulation in neuropathic mice was not observed in
$\mathrm{Cg} 1^{\mathrm{Glu}}$, but remained in $\mathrm{Cg} 2^{\text {Glu }}$ (Fig. $1 D$; paired $t$ test, pinch vs pre, $p=0.7091$ and $<0.001$, respectively). The responses of $\mathrm{Cg} 2^{\text {Glu }}$ to painful stimulation were further verified by measuring their calcium signals in freely moving mice. pAOV-CaMKII $\alpha$ GCAMP6(s)-eYFP was infused into MCC Cg2 to transfect glutamatergic neurons (Fig. 1E). The specificity of virus targeting was confirmed by the colocalization of GCaMP6s-eYFP with 
CaMKII $\alpha$, a marker for glutamatergic neurons (Fig. $1 F$ ). We found that noxious pinch stimulation evoked an immediate withdrawal response but a hardly detectable change in neuronal calcium signals. Considering the pinch stimulation is too brief to elicit remarkable changes, we then measured the calcium signals during persistent pain induced by formalin. The calcium signals of $\mathrm{Cg} 2{ }^{\mathrm{Glu}}$ in the left MCC dramatically reduced immediately after the injection of formalin, but not saline in the right hindpaw. The reduction was long-lasting and did not rebound at the end of $1 \mathrm{~h}$ observation (Fig. $1 G, H$ ). Consistently, the average firing frequency of these neurons decreased lastingly after the injection of formalin (Fig. 1I). These results demonstrate that MCC Cg2 Glu reduce their activity upon noxious stimulation and become hypoactive under neuropathic condition.

MCC Cg2 ${ }^{\text {Glu }}$ activation negatively modulates pain perception The modulatory role of MCC $\mathrm{Cg} 2{ }^{\mathrm{Glu}}$ in nociception was then tested by optogenetic manipulation of their activity. ChR2 virus or Arch virus selectively targeting glutamatergic neurons was infused into the $\mathrm{Cg} 2$ (Fig. 2A). The precise expressions of viruses were confirmed by the colocalization of eYFP with CaMKII $\alpha$ and faithful neuronal responses to laser illumination at the MCC Cg2 (Fig. 2B,C,I). We found that optical activation of unilateral $\mathrm{Cg} 2{ }^{\text {Glu }}$ using blue laser in mice receiving ChR2 virus significantly inhibited mechanical nociception in bilateral hindpaws, as indicated by the increased PWT and PWL (Fig. 2D,E) without interaction of laser stimulation and side of testing (two-way ANOVA with repeated measures and Bonferroni's post-test; Fig. 2D: $F_{(1,18)}=0.3391, p=0.5676$, laser on vs laser off, $p=0.049$ and 0.0058 for ipsilateral and contralateral, respectively; $2 E: F_{(1,18)}=$ $0.5890, p=0.4528$, laser on vs laser off, $p=0.0171$ and 0.0015 for ipsilateral and contralateral, respectively). Interestingly, thermal nociception measured by hotplate test (Fig. 2F; unpaired $t$ test, $p=0.5838)$ and Hargreaves test was not changed by activating $\mathrm{Cg} 2{ }^{\text {Glu }}$ (Fig. 2G; two-way ANOVA with repeated measures and Bonferroni's post-test: $F_{(1,18)}=0.02077, p=0.887$, laser on vs laser off, $p>0.9999$ for ipsilateral and contralateral). In addition, activation of $\mathrm{Cg} 2$ Glu also significantly reduced the duration of painful responses in Phase 2 of the formalin test in the contralateral hindpaw, demonstrating an inhibitory effect on inflammatory pain (Fig. $2 H$; unpaired $t$ test, $p<0.001$ ). These results suggest that MCC $\mathrm{Cg} 2$ Glu activation negatively modulates pain perception in a modality-dependent manner.

We then studied whether $\mathrm{Cg} 2{ }^{\text {Glu }}$ play a tonic role in pain modulation by optically silencing them with yellow laser in mice receiving Arch virus. We found that optical inhibition of $\mathrm{Cg} 2 \mathrm{Glu}$ decreased the contralateral PWT (Fig. 2J; two-way ANOVA with repeated measures and Bonferroni's post-test: $F_{(1,18)}=5.418$, $p=0.0318$, laser on vs laser off, $p=0.7456$ and $<0.001$, respectively, for ipsilateral and contralateral) without effect on thermal nociception (Fig. $2 K$; hotplate test: unpaired $t$ test, $p=0.9373 ; 2 L$; Hargreaves test: two-way ANOVA with repeated measures and Bonferroni's post-test: $F_{(1,18)}=0.487, p=0.4942$, laser on vs laser off, $p>0.9999$ for ipsilateral, $p=0.5474$ for contralateral), suggesting that MCC Cg2 ${ }^{\text {Glu }}$ tonically inhibit nociception and constitute a component of the intrinsic pain inhibitory circuitry.

\section{Projections to the $\mathrm{ZI}^{\mathrm{GABA}}$ contribute the pain inhibitory role of MCC $\mathrm{Cg} 2$ Glu}

To explore whether the pain modulation by MCC Cg2 ${ }^{\mathrm{Glu}}$ is performed by influencing neurons nearby, CNQX and AP5 were infused into $\mathrm{Cg} 2$ to block local glutamatergic neurotransmission (Fig. 3A). We found that the inhibitory effect of optical activation of $\mathrm{Cg} 2{ }^{\text {Glu }}$ on mechanical nociception (Fig. $3 B$; unpaired $t$ test; in saline group: $p=0.0039$ and 0.0066 for ipsilateral and contralateral, respectively; in CNQX + AP5 group: $p=0.0049$ and $<0.001$ for ipsilateral and contralateral, respectively) and pain responses in Phase 2 of the formalin test remained (Fig. $3 C$; unpaired $t$ test, $p=0.0021$ and $<0.001$ in saline and CNQX + AP5 groups, respectively). These results suggest that the pain modulatory function of MCC $\mathrm{Cg} 2{ }^{\text {Glu }}$ is mediated by projecting to other brain regions, but not by acting on neighboring neurons.

To unravel the targets of MCC $\mathrm{Cg} 2{ }^{\text {Glu }}$ projections, we mapped the distribution of eYFP carried by the virus targeting MCC Cg2 ${ }^{\text {Glu }}$. EYFP-positive axons were observed in several ipsilateral brain regions, including the cortex, thalamus, and brainstem, with intensive projections in the $\mathrm{ZI}$, mediorostral part of lateral posterior thalamic nucleus, DLPAG, and retrosplenial granular cortex. These findings coincided with those of Fillinger et al. (2018) who mapped the efferents of 24a' (MCC Cg2) of the mouse.

We noticed that projections to the ZI were highly convergent in the rostral and dorsal subdivisions and less in the ventral subdivision (Fig. 3D). Thus, the role of the MCC Cg2 ${ }^{\text {Glu }}$ to $\mathrm{ZI}$ circuit in pain modulation was first dissected by activating $\mathrm{Cg} 2{ }^{\mathrm{Glu}}$ terminals in the ZI. In mice receiving ChR2 virus infusion into $\mathrm{Cg} 2$, illumination of the ZI terminals by blue laser significantly inhibited mechanical nociception (Fig. 3E; two-way ANOVA with repeated measures and Bonferroni's post-test: $F_{(1,17)}=3.522, p=$ 0.0778 ; laser on vs laser off, $p>0.9999$ for ipsilateral and $=$ 0.0417 for contralateral) as well as painful responses in Phase 2 of the formalin test contralaterally in a glutamate-dependent manner (Fig. 3F-H; unpaired $t$ test, von Frey test: $p<0.001$ and $=$ 0.2418 in saline and CNQX + AP5 groups, respectively; in Phase 2 of formalin test: $p=0.0067$ and 0.6334 for laser on saline vs laser off and laser on CNQX+AP5 vs laser off, respectively). These results demonstrate that projections to the $\mathrm{ZI}$ contribute the pain modulatory role of MCC $\mathrm{Cg} 2{ }^{\text {Glu }}$. MCC $\mathrm{Cg} 2{ }^{\text {Glu }}$ also send dense projections to the DLPAG (Fig. 3I). However, optical activation of $\mathrm{Cg} 2{ }^{\text {Glu }}$ terminals in the DLPAG did not affect mechanical nociception (Fig. 3J; two-way ANOVA with repeated measures and Bonferroni's post-test: $F_{(1,17)}=0.1069, p=0.7477$, laser on vs laser off, $p>0.9999$ for ipsilateral and contralateral), suggesting that projections to the DLPAG are not significantly involved in the pain inhibitory function of MCC $\mathrm{Cg} 2$ Glu.

Given the ZI comprises mainly GABAergic neurons (Chou et al., 2018), the functional and synaptic connection of MCC $\mathrm{Cg} 2^{\text {Glu }}$ terminals with $\mathrm{ZI}^{\mathrm{GABA}}$ were further studied. Single-unit recordings found that the majority of $\mathrm{ZI}$ neurons (59\%) were activated by optical activation of $\mathrm{Cg} 2{ }^{\text {Glu }}$ terminals in the ZI (Fig. $4 A$ ). Retrograde trans-synaptic tracing of $\mathrm{ZI}^{\mathrm{GABA}}$ revealed that presynaptic neurons were located in both $\mathrm{Cg} 2$ and $\mathrm{Cg} 1$ of MCC with a higher number in the former (Fig. $4 B, C$; unpaired $t$ test, $p=0.0362$ ). There neurons were also labeled with CaMKII $\alpha$ (Fig. $4 D$ ), suggesting that more $\mathrm{Cg} 2{ }^{\text {Glu }}$ form synaptic connection with $\mathrm{ZI}^{\mathrm{GABA}}$ compared with Cg1 ${ }^{\text {Glu }}$. Moreover, to verify activation of ZI ${ }^{\text {GABA }}$ negatively modulates nociception, $\mathrm{ZI}{ }^{\text {GABA }}$ were optically activated in Vgat-ChR2-eYFP mice (GABAergic neurons in the brain express ChR2) and were inhibited in Vgat-cre mice receiving Arch virus infusion in the ZI, respectively. We found that selective activation and inhibition of $\mathrm{ZI}{ }^{\mathrm{GABA}}$ inhibited and enhanced mechanical nociception in the contralateral hindpaw, respectively (Fig. 4E,F; unpaired $t$ test, $p=0.0388$ and $<0.001$, respectively). The mediating role of $\mathrm{ZI}^{\mathrm{GABA}}$ in pain modulation of MCC $\mathrm{Cg} 2{ }^{\text {Glu }}$ was further confirmed by selectively inhibiting $\mathrm{ZI}^{\text {GABA }}$ by chemogenetic approach and optically activating ZI 
A

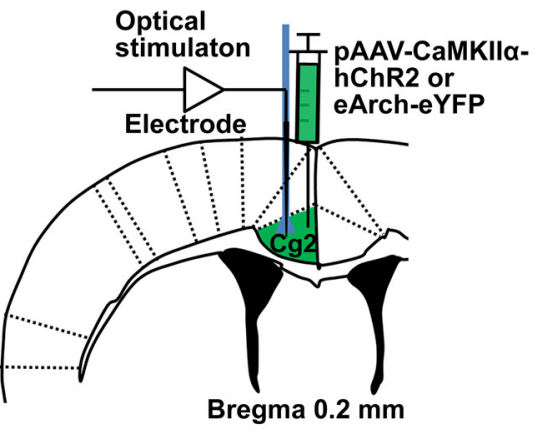

D

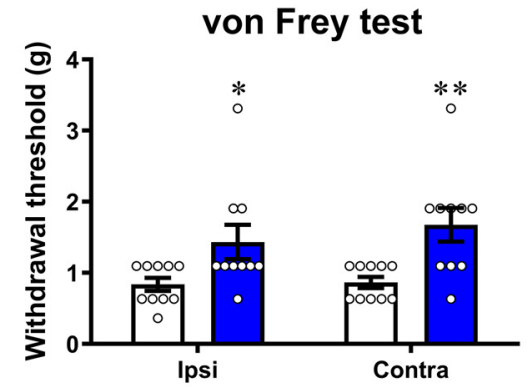

G

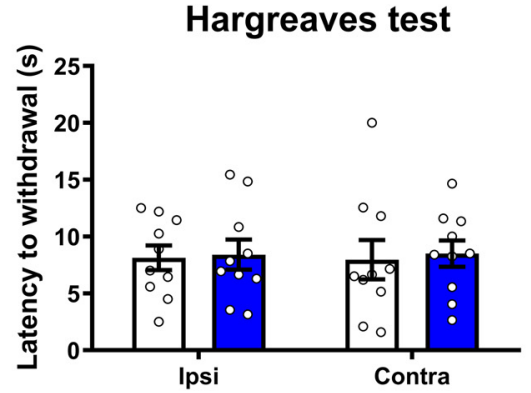

J

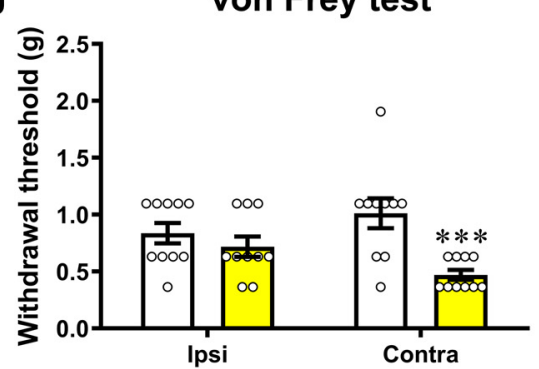

B
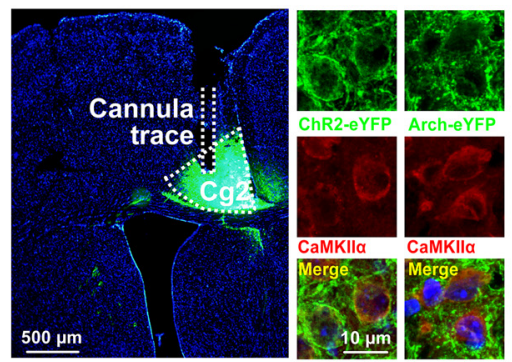

E

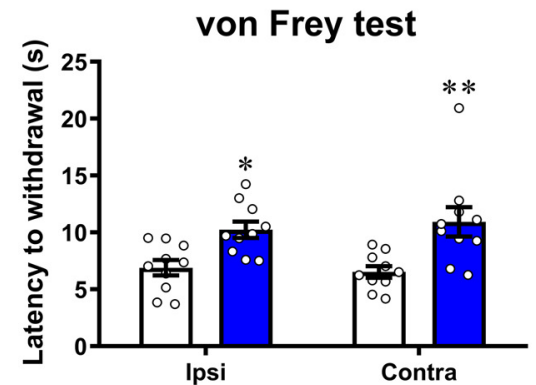

H

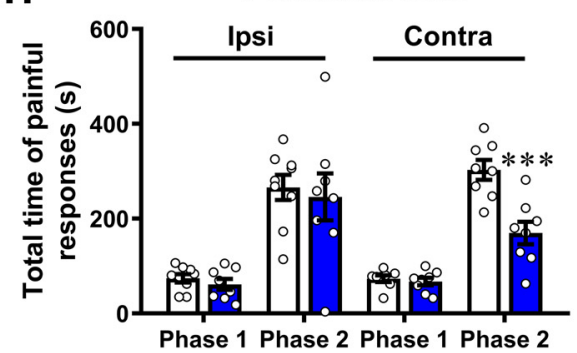

K

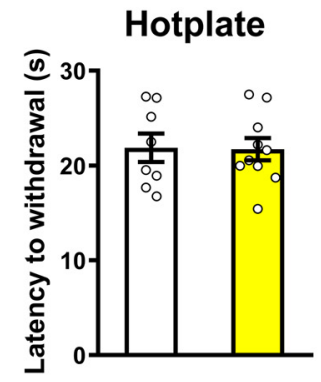

$\mathbf{L}$
C
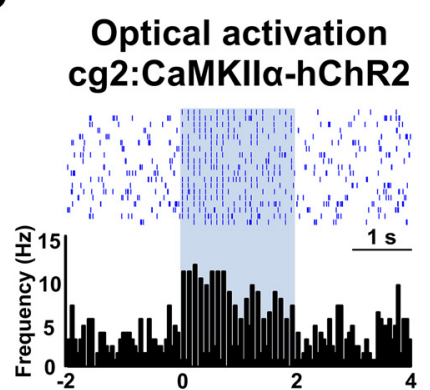

$\mathbf{F}$

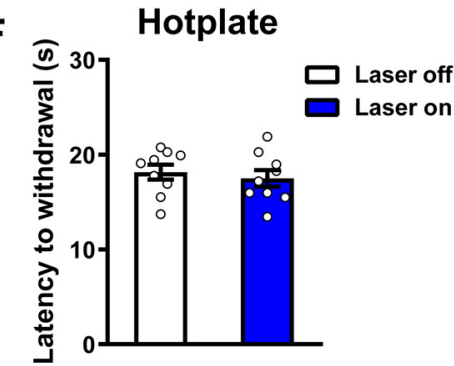

I
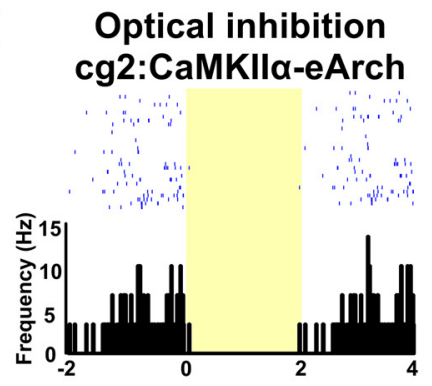

Hargreaves test

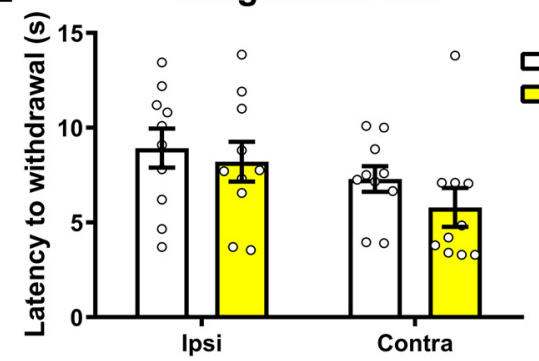

Figure 2. $\quad \mathrm{MCC} C \mathrm{Cg}{ }^{\text {Glu }}$ negatively modulate pain perception. $A$, Schematic drawing of the sites for virus injection, and recording electrode or cannula placement. $\boldsymbol{B}$, Example photomicrographs showing the colocalization of ChR2-eYFP or Arch-eYFP with CaMKII $\alpha$ in MCC Cg2.C, Example neuron in MCC Cg2 that increased its firing frequency in response to blue $(10 \mathrm{~Hz}, 10 \mathrm{~ms}, 5 \mathrm{~mW}, 2 \mathrm{~s}$ on/off cycle) laser stimulation. D, Optical activation of unilateral MCC $\mathrm{Cg} 2{ }^{\text {Glu }}$ elevated PWT bilaterally in intact mice. $n=10 /$ group, two-way ANOVA with repeated measures and Bonferroni's post-test $\left(F_{(1,18)}=0.3391, p=0.5676\right)$, laser on versus laser off, ipsilateral (ipsi), $p=0.049$; contralateral (contra), $p=0.0058$. E, Optical activation of unilateral MCC Cg2 $2^{\text {Glu }}$ prolonged PWL bilaterally. $n=$ 10/group, two-way ANOVA with repeated measures and Bonferroni's post-test $\left(F_{(1,18)}=0.5890, p=0.4528\right)$, laser on versus laser off, ipsilateral, $p=0.0171 ;$ contralateral, $p=0.0015 . \boldsymbol{F}, \mathbf{G}$, Optical activation of unilateral $\mathrm{MCC} \mathrm{Cg}{ }^{\text {llu }}$ did not affect thermal sensitivity in hotplate test $(\boldsymbol{F})$ and Hargreaves test $(\boldsymbol{G})$ in intact mice. Hotplate test: $n=9 /$ group, two-tailed unpaired $t$ test, laser on versus laser off, $p=0.5838, t=0.5592, \mathrm{df}=16$. Hargreaves test: $n=10 /$ group, two-way ANOVA with repeated measures and Bonferroni's post-test $\left(F_{(1,18)}=0.02077, p=0.887\right)$, laser on versus laser off, ipsilateral and contralateral, $p>0.9999$. $\boldsymbol{H}$, Optical activation of unilateral $\mathrm{MCC} \mathrm{Cg}^{\text {Glu }}$ reduced formalin-induced pain contralaterally, but not ipsilaterally. In Phase 2, two-tailed unpaired $t$ test, laser on versus laser off. Ipsilateral, laser off $n=9$, laser on $n=8, p=0.7162, t=0.3705$, df $=15$; contralateral, $n=8 /$ group, $p<0.001, t=4.201$, df $=14$. I, Example neuron in MCC Cg2 that deceased its firing frequency in response to yellow (direct current, $5 \mathrm{~mW}, 2 \mathrm{~s}$ on/off cycle) laser stimulation.J, Optical inhibition of unilateral MCc C $\mathrm{g} 22^{\mathrm{Glu}}$ reduced PWT contralaterally in intact mice. $n=10 /$ group, two-way ANOVA with repeated measures and Bonferroni's post-test $\left(F_{(1,18)}=5.418, p=0.0318\right)$, laser on versus laser off, ipsilateral, $p=0.7456 ;$ contralateral, $p<$ 0.001 . $K, L, O p t i c a l$ inhibition of unilateral $\mathrm{MCC}\left(\mathrm{g} 2{ }^{\text {Glu }}\right.$ had no effect on thermal sensitivity in both hotplate test $(\boldsymbol{K})$ and Hargreaves test $(\boldsymbol{L})$ in intact mice. Hotplate test: laser off $n=8$, laser on $n=$ 10, two-tailed unpaired $t$ test, laser on versus laser off, $p=0.9373, t=0.07991, \mathrm{df}=16$. Hargreaves test: $n=10$ /group, two-way ANOVA with repeated measures and Bonferroni's post-test $\left(F_{(1,18)}=0.487, p=0.4942\right)$, laser on versus laser off, ipsilateral, $p>0.9999 ;$ contralateral, $p=0.5474 .{ }^{*} p<0.05,{ }^{* *} p<0.01,{ }^{* * *} p<0.001$.

terminals of MCC $\mathrm{Cg} 2{ }^{\mathrm{Glu}}$ simultaneously. $\mathrm{CNO}(1 \mathrm{mg} / \mathrm{kg}$, i.p.) abrogated the pain inhibitory effect of activating MCC $\mathrm{Cg}_{2}{ }^{\mathrm{Glu}}$ in the mice receiving virus carrying hM4Di targeting GABAergic neurons (Fig. 4H; one-way ANOVA with Tukey's post hoc:
$F_{(3,24)}=7.872, p<0.001$; laser on vs laser off, $p=0.0025$, laser on + hM4Di + CNO vs laser on, $p=0.0048)$. These results demonstrate that the excitatory innervation of $\mathrm{ZI}^{\mathrm{GABA}}$ by MCC $\mathrm{Cg} 2{ }^{\text {Glu }}$ negatively modulates nociception. 
A

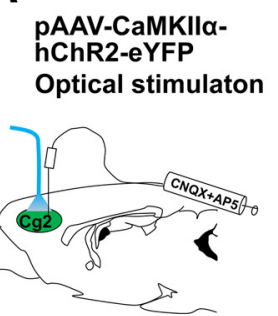

D

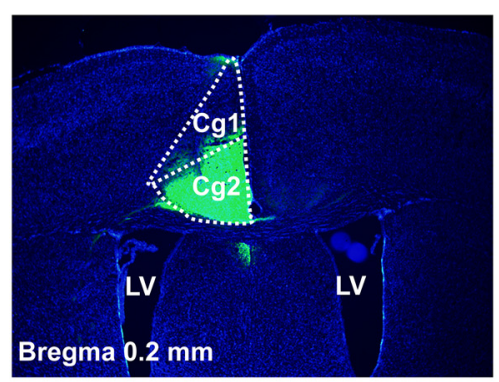

F

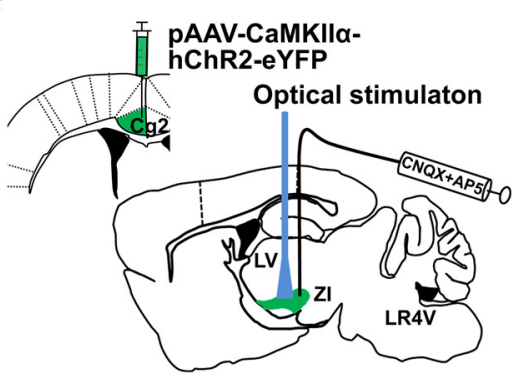

I

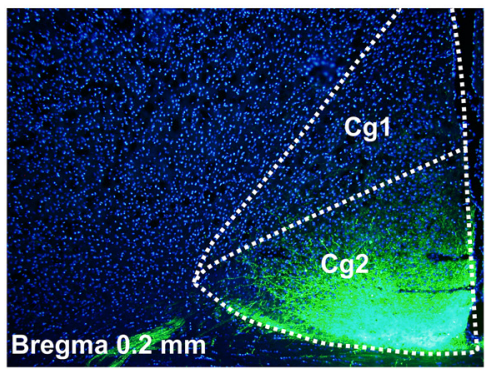

B

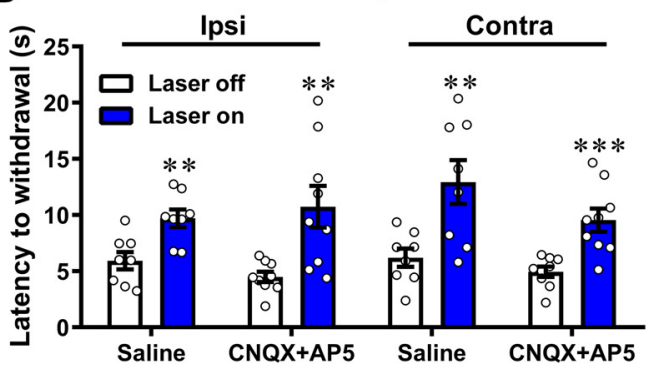

C

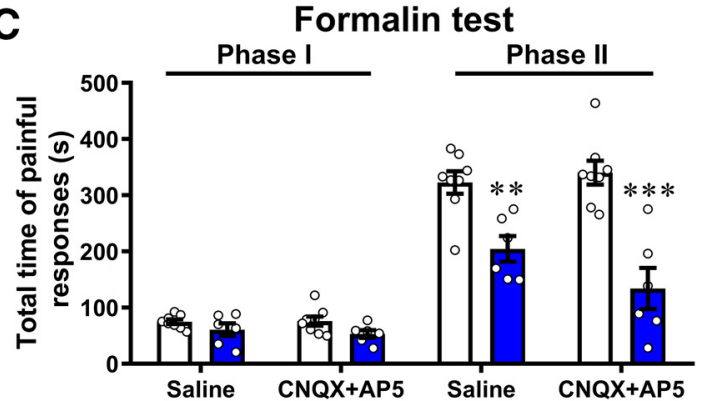

E

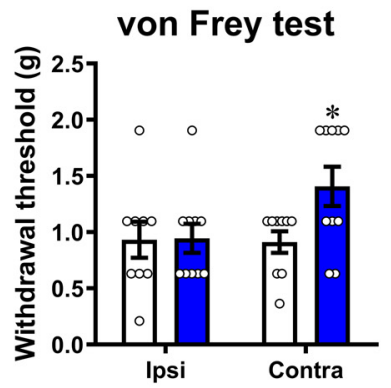

G von Frey test
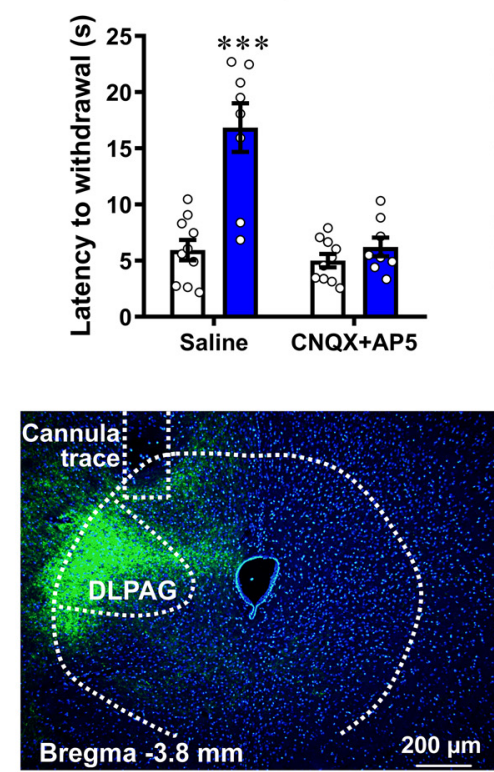

H

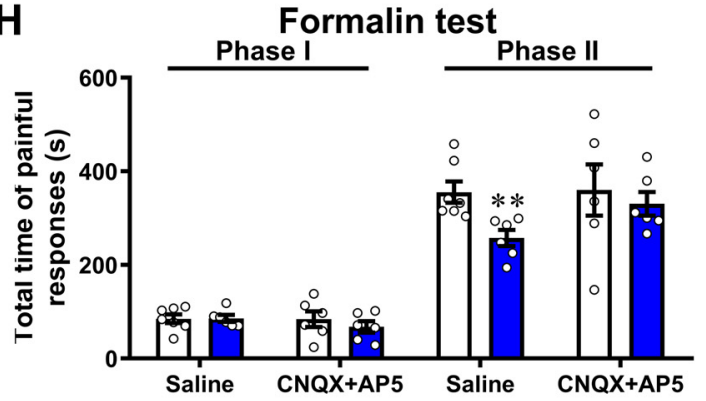

J

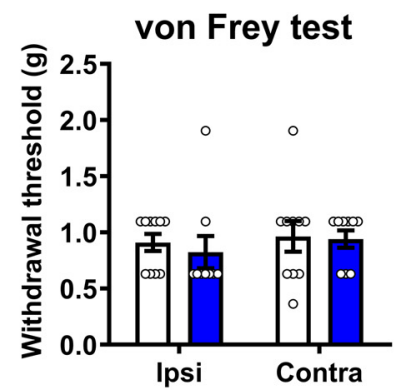

Figure 3. Projections to Zl mediate the pain inhibitory function of $M C C \mathrm{Cg}{ }^{\text {Glu }}$. $\boldsymbol{A}$, Schematic drawing of optical activation and administration of CNQX and AP5. $\boldsymbol{B}$, CNQX and AP5 did not affect the pain inhibitory effect of optically activating MCCCg2 ${ }^{\text {Glu }}$ in the von Frey test. Saline $n=8 /$ group, CNQX $+A P 5 n=9 /$ group, two-tailed unpaired $t$ test, laser on versus laser off. Ipsilateral: saline, $p=0.0039, t=3.448$, $\mathrm{df}=14 ; \mathrm{CNQX}+\mathrm{AP5}, p=0.0049, t=3.262, \mathrm{df}=16$. Contralateral: saline, $p=0.0066, t=3.187, \mathrm{df}=14 ; \mathrm{CNQX}+\mathrm{AP5}, p<0.001, t=4.085, \mathrm{df}=16 . \mathrm{C}, \mathrm{CNQX}$ and AP5 did not affect the pain inhibitory effect of optically activating MCC $\mathrm{Cg} 2$ Glu in the formalin test. Laser off $n=8 /$ group, laser on $n=6 /$ group, two-tailed unpaired $t$ test, laser on versus laser off. In Phase $2:$ saline, $p=0.0021, t=3.913$, df $=12$; CNQX +AP5, $p<0.001, t=5.153, \mathrm{df}=12 . \boldsymbol{D}$, Example photomicrographs for ChR2-eYFP expression in the MCC Cg2 (left) and ZI (right). Scale bar, $500 \mu m$ m. $\boldsymbol{E}, 0$ ptical activation of MCC Cg2 ${ }^{\text {Glu }}$ terminals in the Zl elevated PWT contralaterally in intact mice. Laser off $n=9$, laser on $n=10$, two- way ANOVA with repeated measures and Bonferroni's post-test $\left(F_{(1,17)}=3.522, p=0.0778\right)$, laser on versus laser off, ipsilateral, $p>0.9999$; contralateral, $p=0.0417$. $\boldsymbol{F}-\boldsymbol{H}$, Administration of CNQX and AP5 in the Zl abrogated the pain inhibitory effect of optically activating MCC $\mathrm{Cg} 2$ Glu terminals in the Zl in the von Frey test (G) and in the formalin test $(\boldsymbol{H})$. von Frey test: laser off $n=10$, laser on $n=8$, two-tailed unpaired $t$ test, laser on saline versus laser off, $p<0.001, t=5.003$, df $=16$; laser on CNQX + AP5 versus laser off, $p=$ $0.2418, t=1.215, \mathrm{df}=16$. Formalin test: saline, laser off $n=7$, laser on $=6 ; \mathrm{CNQX}+\mathrm{AP5}, n=6 /$ group, two-tailed unpaired $t$ test. In Phase 2, laser on saline versus laser off, $p=0.0067, t=3.330, \mathrm{df}=$ 11 ; laser on CNQX + AP5 versus laser off, $p=0.6334, t=0.4920, \mathrm{df}=10 . I$, Example photomicrographs for ChR2-eYFP expression in MCC Cg2 and DLPAG, scale bar, $200 \mu \mathrm{m}$. J, Optical activation of DLPAG terminals of MCC Cg2 ${ }^{\text {Glu }}$ did not affect PWT in intact mice. Laser off $n=10$, laser on $n=9$, two-way ANOVA with repeated measures and Bonferroni's post-test $\left(F_{(1,17)}=0.1069, p=0.7477\right)$, laser on versus laser off, $p>0.9999$ for ipsilateral and contralateral. ${ }^{*} p<0.05,{ }^{* *} p<0.01,{ }^{* * *} p<0.001$.

Consecutive activation of MCC Cg2 ${ }^{\text {Glu }}$ alleviates neuropathic pain

To study the contribution of hypoactive MCC Cg2 ${ }^{\text {Glu }}$ to neuropathic hypersensitivity, the effects of optogenetic manipulation of these neurons on mechanical allodynia were tested on D7 and D14 following PSL when the mechanical threshold decreased sig- nificantly in bilateral hindpaws. In neuropathic mice receiving $\mathrm{ChR} 2$ virus targeting MCC Cg2 Glu, we found that only daily photoactivation of these neurons through postoperative days resulted in an attenuation of mechanical allodynia in the contralateral hindpaw (the nerve-injured side), but the brief photoactivation on test days, which produced an instant pain inhib- 
A
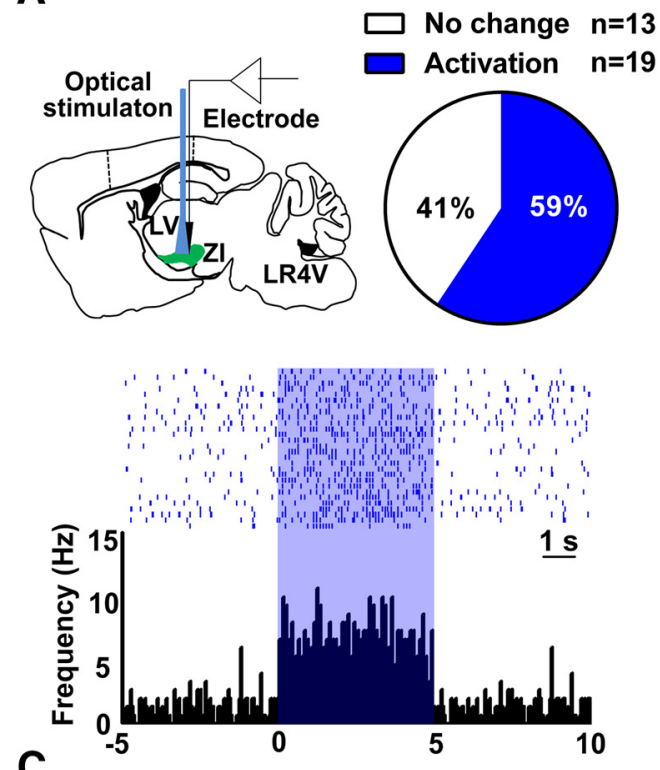

C

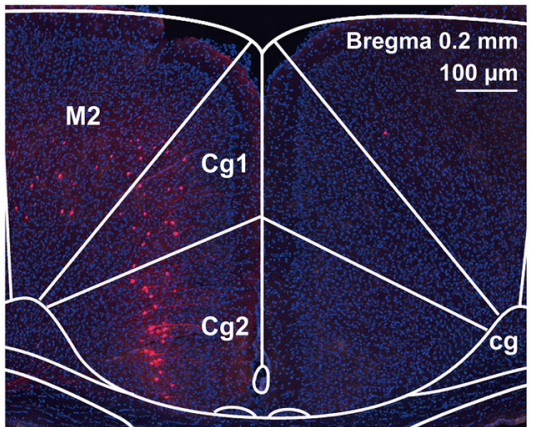

\section{B}
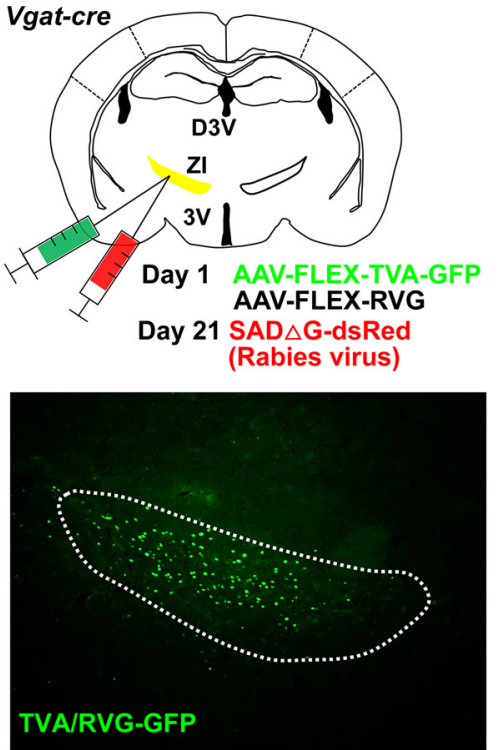

Merge
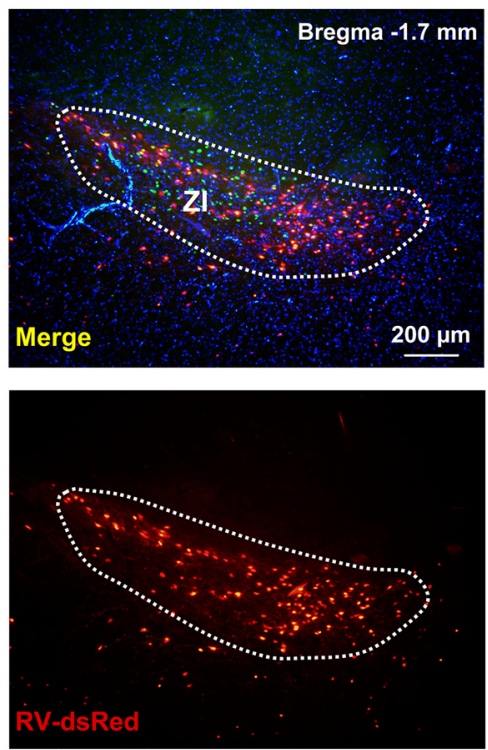

D
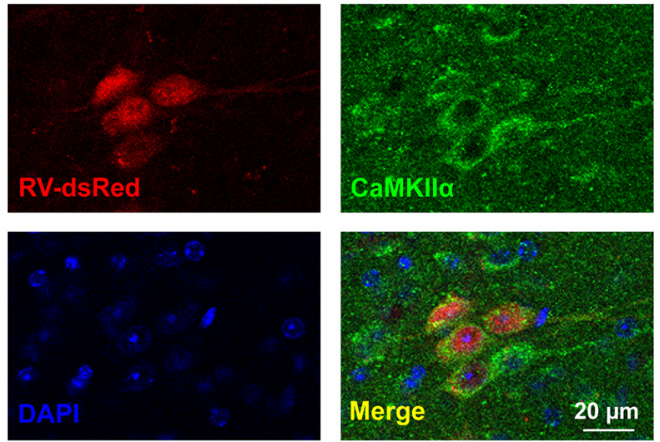

E

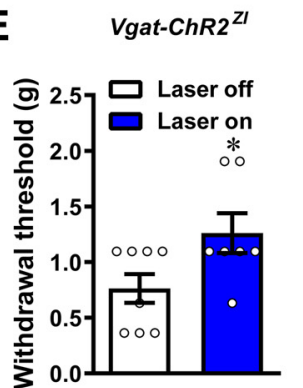

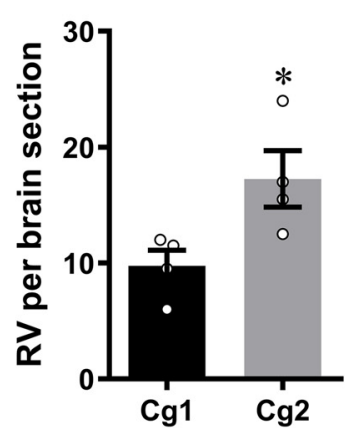

G

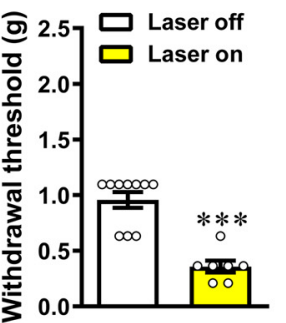

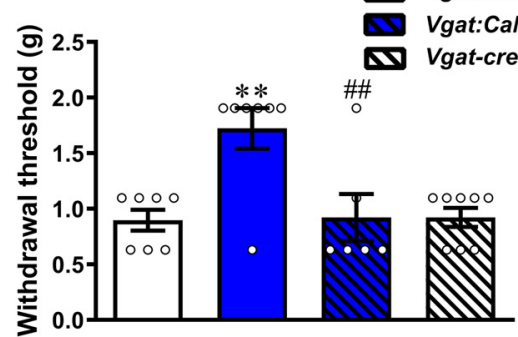

Figure 4. $\mathrm{MCC} C \mathrm{Cg} 2^{\text {Glu }}$ inhibit pain perception by activating ZI ${ }^{\mathrm{GABA}} \cdot A$, Schematic drawing of in vivo single-unit recording of ZI neurons during optical activation of Zl terminals of $\mathrm{MCC} C \mathrm{Cg} 2{ }^{\text {Glu }}$ (top left), percentage of neurons that increased or remained their activity upon optical activation (top right) and example neuron that was activated by illumination (bottom). $\boldsymbol{B}$, Schematic drawing of virus injection for trans-synaptic tracing of presynaptic inputs to ZI ${ }^{\mathrm{GABA}}$ (upper left) and representative images of RV-infected starter neurons in the Zl (top right and bottom). C, Representative image of trans-synaptically labeled neurons in the MCC (left) and the numbers of RV-infected neurons in Cg2 (gray) and Cg1 (black; right). $n=4$, two-tailed unpaired $t$ test, $\mathrm{Cg} 2$ versus Cg1, $t=2.687$, df $=$ $6, p=0.0362$. D, Photomicrographs showing trans-synaptically labeled neurons by RV in MCC $\mathrm{Cg} 2$ were colabeled with CaMKIl $\alpha$. E, Optical activation of ZI ${ }^{\mathrm{GABA}}$ elevated the contralateral PWT in intact Vgat-ChR2 mice. Laser off $n=8$, laser on $n=7$, two-tailed unpaired $t$ test, laser on versus laser off, $p=0.0388, t=2.298, \mathrm{df}=13$. F, Optical inhibition of Zl ${ }^{\text {GABA }}$ reduced the contralateral PWT in intact Vgat-Cre mice. Laser off $n=10$, laser on $n=7$, two-tailed unpaired $t$ test, laser on versus laser off, $p<0.001, t=6.221$, df $=15.6$, hM4Di-mediated inhibition of Zl ${ }^{\text {GABA }}$ reversed the pain inhibitory effect of optically activating Zl terminals of $\mathrm{MCC} C \mathrm{Cg}^{\text {Glu }}$. Laser off $n=7$, laser on $n=7$, laser on $+\mathrm{hM4Di}+\mathrm{CNO} n=6, \mathrm{CNO} n=8.0$ ne-way ANOVA with Tukey's posthoc $\left(F_{(3,24)}=7.872\right.$, $p<0.001)$, laser on versus laser off, $p=0.0025$, laser on $+\mathrm{hM} 4 \mathrm{Di}+\mathrm{CNO}$ versus laser on, $p=0.0048$. ${ }^{*} p<0.05$, Cg2 versus Cg1 in C, laser on versus laser off in $\boldsymbol{E}$. ${ }^{* *} p<0.01$, ${ }_{* * *} p<0.001$, laser on versus laser off. \#\#p $<0.01$, laser on $+\mathrm{hM4Di}+$ CNO versus laser on.

itory effect in intact mice, did not (Fig. $5 A$; one-way ANOVA with Tukey's post hoc; D7: $F_{(3,32)}=18.69, p<0.001$, laser off vs sham, $p<0.001$, laser on daily vs laser off, $p=0.0202$; D14: $F_{(3,32)}=$ $18.01, p<0.001$, laser off vs sham, $p<0.001$, laser on daily vs laser off, $p=0.0023$ ). These results suggest that the hypoactivity of MCC Cg2 $2^{\text {Glu }}$ in neuropathy diminishes their pain inhibitory strength and consecutive activation is required to compensate this reduction.

It is known that clinical patients with neuropathic conditions commonly suffer spontaneous ongoing pain. We further evaluated the effect of MCC $\mathrm{Cg} 2$ Glu activation on spontaneous ongoing pain by the CPP test, in which animals with ongoing pain will 
A

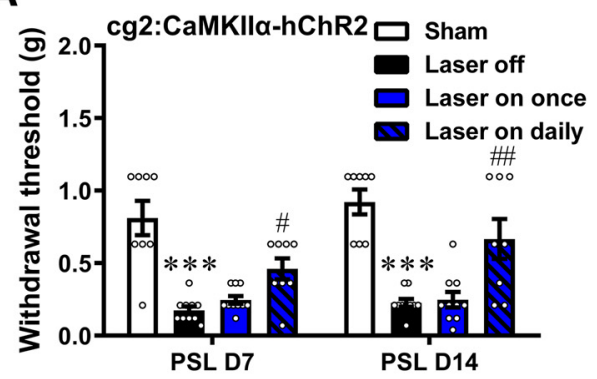

C

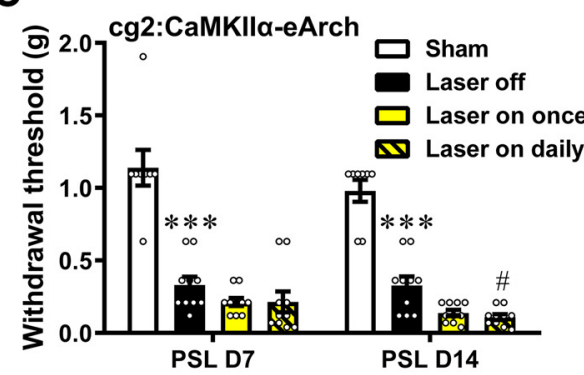

E

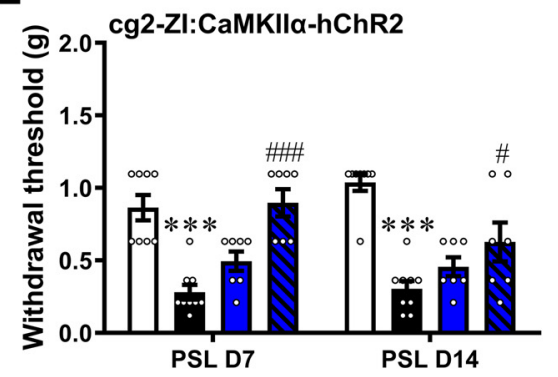

G

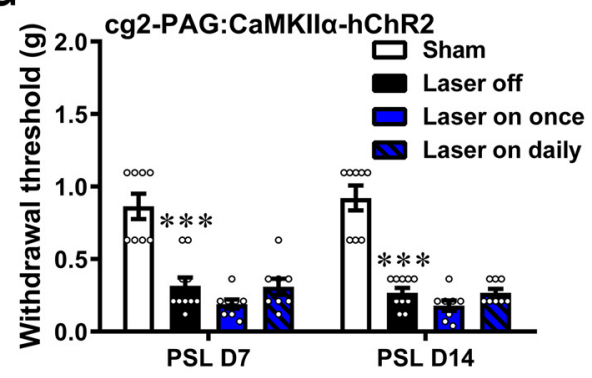

B
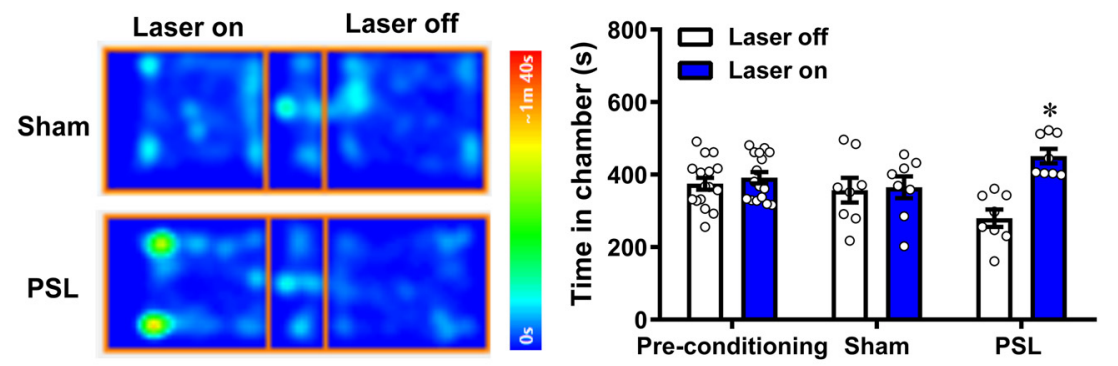

D
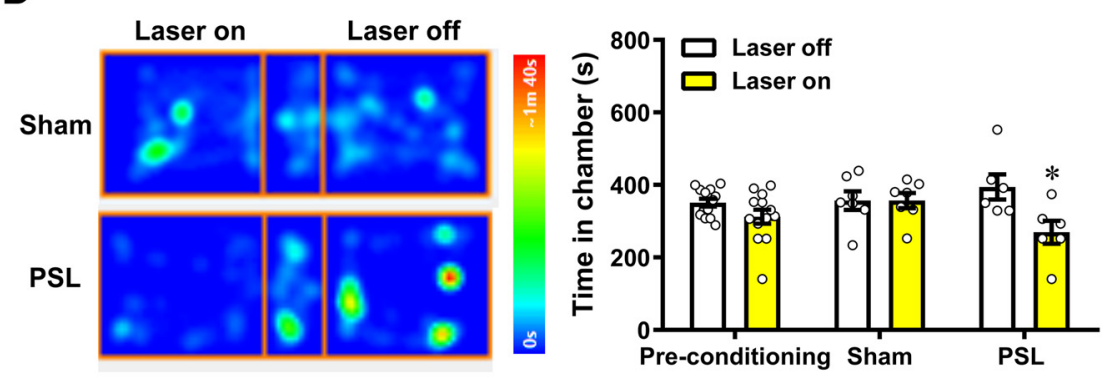

F
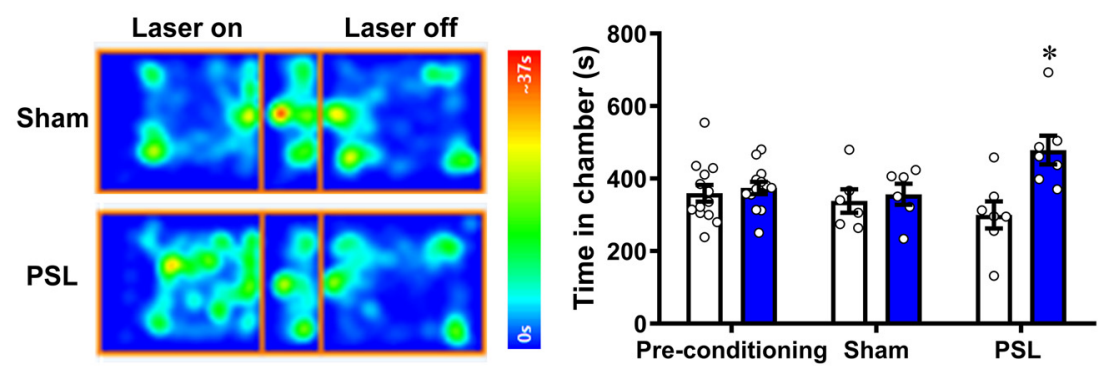

H
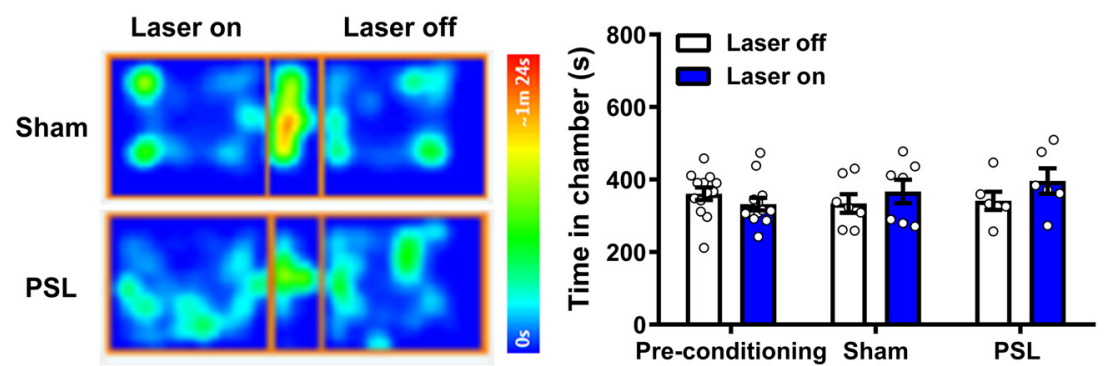

Figure 5. Activation of $\mathrm{MCC} C \mathrm{Cg}{ }^{\mathrm{Glu}}$ to $\mathrm{Zl}{ }^{\mathrm{GABA}}$ circuit alleviates neuropathic pain. $A, 0$ ptical activation of unilateral $\mathrm{MCC} \mathrm{Cg} 2{ }^{\text {Glu }}$ alleviated neuropathic allodynia contralaterally. Sham and laser on daily $n=$ 8/group, laser off and laser on once $n=10 /$ group, one-way ANOVA with Tukey's post hoc; $\mathrm{D7}: \mathrm{F}_{(3,32)}=18.69, p<0.001$, laser off versus sham, $p<0.001$, laser on daily versus laser off, $p=0.0202 ; \mathrm{D} 14$ : $F_{(3,32)}=18.01, p<0.001$, laser offversus sham, $p<0.001$, laser on daily versus laser off, $p=0.0023 . B, 0$ ptical activation of unilateral MCC $\mathrm{Gg} 2^{\text {Glu }}$ induced place preference in neuropathic mice. $n=8 /$ group, two-way ANOVA with repeated measures and Bonferroni's post-test $\left(F_{(2,58)}=7.314, p=0.0015\right)$. PSL group, testversus preconditioning, $p=0.0219$. C, Optical inhibition of unilateral MCC $\mathrm{Cg}{ }^{\text {Glu }}$ deteriorated neuropathic allodynia contralaterally. Sham $n=8$, laser off, laser on once and laser on daily $n=10$ /group, one-way ANOVA with Tukey's post hoc; D7: $F_{(3,34)}=34.06, p<0.001$, laser off versus sham, $p<$ 0.001 , laser on daily versus laser off, $p=0.6463 ; \mathrm{D14}: F_{(3,34)}=75.97, p<0.001$, laser offversus sham, $p<0.001$, laser on daily versus laser off, $p=0.0315 . D, 0$ ptical inhibition of unilateral MCC $\mathrm{Cg} 2$ Glu induced place averseness in neuropathic mice. Sham $n=7, \mathrm{PSL} n=6$, two-way ANOVA with repeated measures and Bonferroni's post-test $\left(F_{(2,46)}=3.214, p=0.0494\right)$. PSL group, test versus preconditioning, $p=$ 0.0122.E, Optical activation of Zl terminals of MCC $C 2^{\text {Glu }}$ alleviated neuropathic allodynia contralaterally. Sham $n=8$, laser off $n=9$, laser on once and laser on daily $n=7 /$ group, one-way ANOVA with Tukey's posthoc; $\mathrm{D7}: F_{(3,27)}=16.45, p<0.001$, laser off versus sham, $p<0.001$, laser on daily versus laser off, $p<0.001 ; \mathrm{D} 14: F_{(3,27)}=16.73, p<0.001$, laser off versus sham, $p<0.001$, laser on daily versus laser off, $p=0.0360 . F$, Optical activation of Zl terminals of MCC $\mathrm{Cg}{ }^{\text {Glu }}$ induced place preference in neuropathic mice. Sham $n=6, \mathrm{PSL} n=7$, two-way ANOVA with repeated measures and Bonferroni's post-test $\left(F_{(2,46)}=5.027, p=0.0106\right)$. PSL group, test versus preconditioning, $p=0.0410$. G, Optical activation of DLPAG terminals of MCC $\mathrm{Cg} 2{ }^{\text {Glu }}$ did not affectneuropathic allodynia. Sham $n=8$, laser off $n=10$, laser on onceand laser on daily $n=8 /$ group, one-way ANOVA with Tukey's posthoc; D7: $F_{(3,30)}=22.90, p<0.001$, laser offversus sham, $p<0.001 ; \mathrm{D} 14: F_{(3,30)}=46.41, p<0.001$, laser offversus sham, $p<0.001$. $\boldsymbol{H}$, Optical activation of DLPAG terminals of MCC Cg2 ${ }^{\text {llu }}$ did not induce place preference in neuropathic mice. Sham $n=7, \operatorname{PSL} n=6$, two-way ANOVA with repeated measures and Bonferroni's post-test $\left(F_{(2,46)}=1.826, p=0.1725\right) .{ }^{*} p<0.05,{ }^{* * *} p<0.001$, laser off versus sham. $\# p<0.05$, \#\#p $<0.01$, \#\#\#p $<0.001$, laser on daily versus laser off.

show preference to the place associated with pain relief (King et al., 2009). We found that neuropathic mice receiving ChR2 virus spent more time in the chamber paired with blue laser stimulation of $\mathrm{Cg} 2{ }^{\mathrm{Glu}}$, demonstrating the relief of spontaneous ongoing pain by photoactivation. Mice in the sham group did not show such a preference, suggesting that MCC $\mathrm{Cg} 2{ }^{\text {Glu }}$ activation does not induce rewarding in animals without ongoing pain (Fig. 5B; two-way ANOVA with repeated measures and Bonferroni's post- 
A

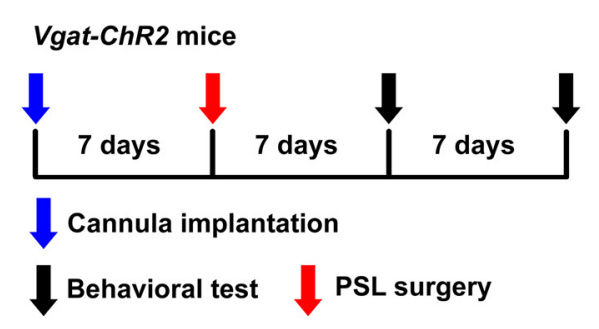

D

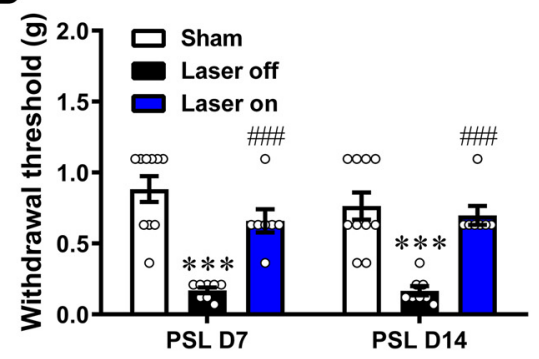

B

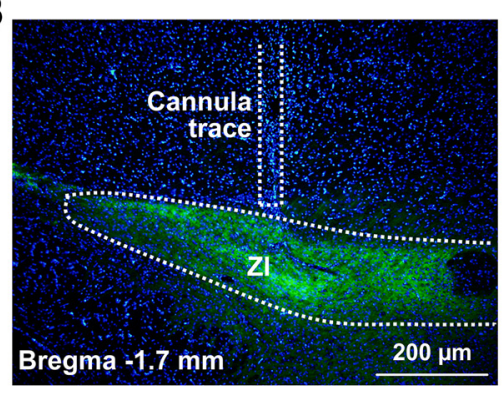

E

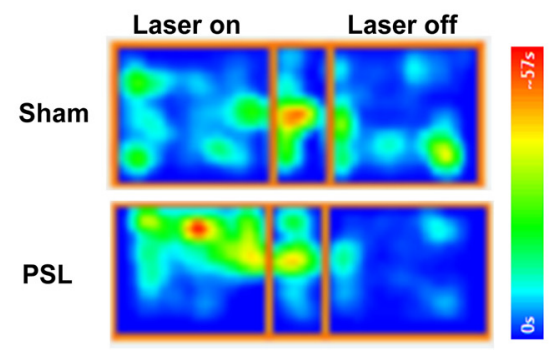

C

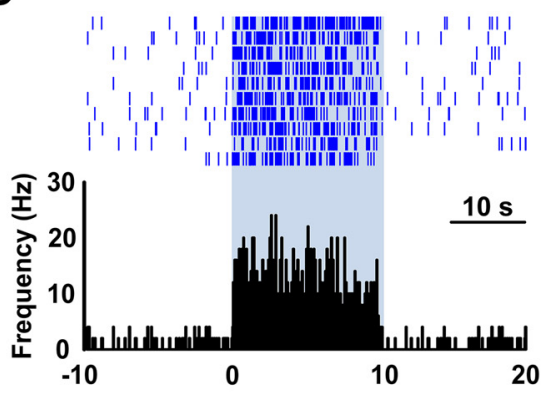

Figure 6. Activation of ZI ${ }^{\text {GABA }}$ alleviates neuropathic pain. $\boldsymbol{A}$, Flow chart of the experiment. $\boldsymbol{B}$, Example photomicrograph for the site of cannula placement. Scale bar, 200 $\mu$ m. $\boldsymbol{C}$, Example ZI neuron that increased its firing frequency in response to blue laser stimulation. $D$, Daily optical activation of unilateral ZI ${ }^{\mathrm{GAA}}$ alleviated neuropathic allodynia contralaterally. Sham $n=10$, laser off $n=8$, laser on $n=7$, one-way ANOVA with Tukey's post hoc; D7: $\left.F_{(2,22)}=24.46, p<0.001\right)$, laser off versus sham, $p<0.001$, laser on versus laser off, $p<0.001 ; D 14: F_{(2,22)}=18.20, p<0.001$, laser off versus sham, $p<0.001$, laser on versus laser off, $p<0.001$. $\boldsymbol{E}, 0$ ptical activation of unilateral ZI ${ }^{\mathrm{GABA}}$ induced place preference in neuropathic mice. Sham $n=7$, PSL $n=10$, tw0-way ANOVA with repeated measures and Bonferroni's post-test $\left(F_{(2,62)}=13.93, p<0.001\right)$. PSL group, test versus preconditioning, $p=0.0012 .{ }^{* *} p<0.01,{ }^{* * *} p<0.001$, laser off versus sham. $\# \#$ \# $<0.001$, laser on versus laser off.

test: $F_{(2,58)}=7.314, p=0.0015$, PSL group, test vs preconditioning, $p=0.0219)$.

The role of MCC Cg2 $2^{\text {Glu }}$ in NeuP was further studied by optically inhibiting their activity in neuropathic mice receiving Arch virus targeting these neurons. On postoperative Day 7, yellow light stimulation given either only once on the test day or daily through postoperative days did not affect the mechanical allodynia. On Day 14, however, daily stimulation through postoperative days worsened mechanical allodynia, but a brief laser stimulation had no effect (Fig. 5C; one-way ANOVA with Tukey's post hoc; D7: $F_{(3,34)}=34.06, p<0.001$, laser off vs sham, $p<0.001$; D14: $F_{(3,34)}=75.97, p<0.001$, laser off vs sham, $p<$ 0.001 , laser on daily vs laser off, $p=0.0315$ ). These results indicate that the tonic pain inhibitory role of MCC $\mathrm{Cg} 2{ }^{\text {Glu }}$ has been greatly impaired so that a consecutive but not a brief or shortterm inhibition of their activity leads to aggravation of neuropathic pain. In the CPP test, neuropathic but not sham-treated mice spent less time in the chamber paired with yellow light stimulation (Fig. 5D; two-way ANOVA with repeated measures and Bonferroni's post-test: $F_{(2,46)}=3.214, p=0.0494$, PSL group, test vs preconditioning, $p=0.0122$ ), indicating that inhibition of MCC $\mathrm{Cg} 2$ Glu activity worsens the aversive affect associated with spontaneous ongoing pain.

Consecutive activation of MCC Cg2 ${ }^{\text {Glu }}$ to $\mathrm{ZI}^{\mathrm{GABA}}$ circuit attenuates neuropathic pain

The function of the MCC Cg2 ${ }^{\text {Glu }}$ to $\mathrm{ZI}^{\mathrm{GABA}}$ circuit in NeuP was examined in neuropathic mice by activating the MCC Cg2 Glu terminals in the ZI. We found that daily photoactivation throughout postoperative days, but not the brief activation on the test day of these terminals alleviated the contralateral mechanical allodynia (Fig. 5E; one-way ANOVA with Tukey's post hoc; D7: $F_{(3,27)}=16.45, p<0.001$, laser off vs sham, $p<0.001$, laser on daily vs laser off, $p<0.001$; D14: $F_{(3,27)}=16.73, p<0.001$, laser off vs sham, $p<0.001$, laser on daily vs laser off, $p=0.036$ ). Moreover, photoactivation induced a preference to light-paired chamber in the CPP test, suggesting the relief of ongoing pain (Fig. 5F; two-way ANOVA with repeated measures and Bonferroni's post-test: $F_{(2,46)}=5.027, p=0.0106$, PSL group, test vs preconditioning, $p=0.041)$. By contrast, consecutive optical activation of MCC Cg2 ${ }^{\text {Glu }}$ terminals in the DLPAG did not affect mechanical allodynia (Fig. 5G; one-way ANOVA with Tukey's post hoc; D7: $F_{(3,30)}=22.90, p<0.001$, laser off vs sham, $p<$ 0.001; D14: $F_{(3,30)}=46.41, p<0.001$, laser off vs sham, $p<$ 0.001 ), nor induce preference in the CPP test (Fig. $5 H$; two-way ANOVA with repeated measures and Bonferroni's post-test: $\left.F_{(2,46)}=1.826, p=0.1725\right)$. These results demonstrate that consecutive activation of the MCC Cg2 ${ }^{\text {Glu }}-\mathrm{ZI}^{\mathrm{GABA}}$ circuit is able to compensate its impaired analgesic function under NeuP condition.

The role of $\mathrm{ZI}^{\mathrm{GABA}}$ in NeuP was examined by activating $\mathrm{ZI}^{\mathrm{GABA}}$ in Vgat-ChR2-eYFP mice (Fig. 6A-C). We found that daily optical activation of $\mathrm{ZI}^{\mathrm{GABA}}$ significantly alleviated mechanical allodynia in the contralateral hindpaw (Fig. 6D; one-way ANOVA with Tukey's post hoc; D7: $F_{(2,22)}=24.46, p<0.001$, laser off vs sham, $p<0.001$, laser on vs laser off, $p<0.001$; D14: $F_{(2,22)}=18.20, p<0.001$, laser off vs sham, $p<0.001$, laser on vs laser off, $p<0.001)$. In the CPP test, blue light illumination of the ZI induced preference to the light-paired chamber in neuropathic mice, suggesting the relief of ongoing pain (Fig. 6E; twoway ANOVA with repeated measures and Bonferroni's post-test: $F_{(2,62)}=13.93, p<0.001$, PSL group, test vs preconditioning, $p=$ $0.0012)$. These results suggest that the pain inhibitory function of $\mathrm{ZI}^{\mathrm{GABA}}$ excitation remains in neuropathy, which guarantees the pain inhibitory modulation by MCC Cg2 ${ }^{\text {Glu }}$ activation. 
A
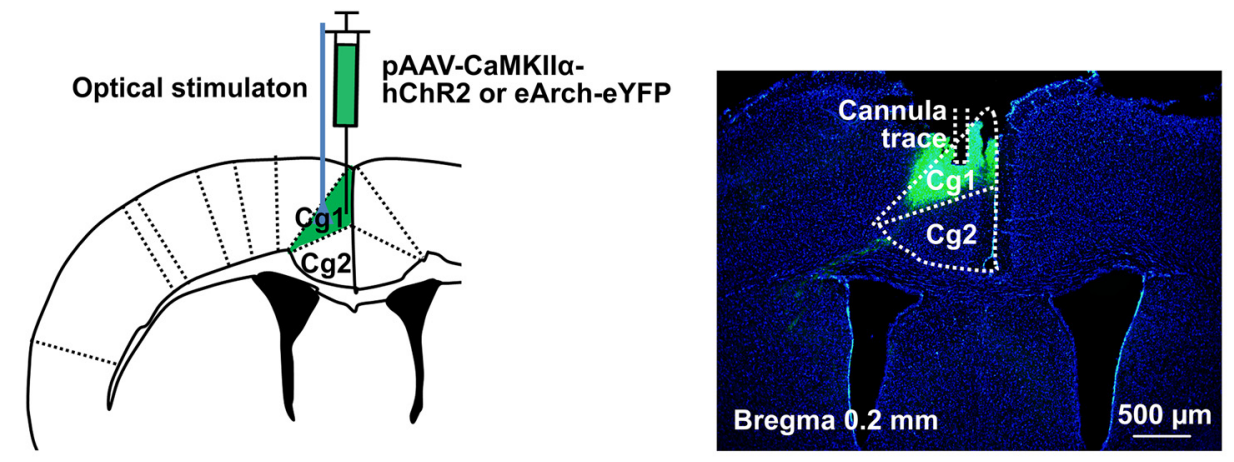

B

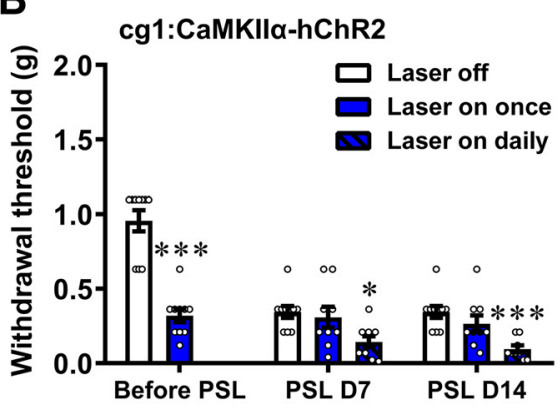

D

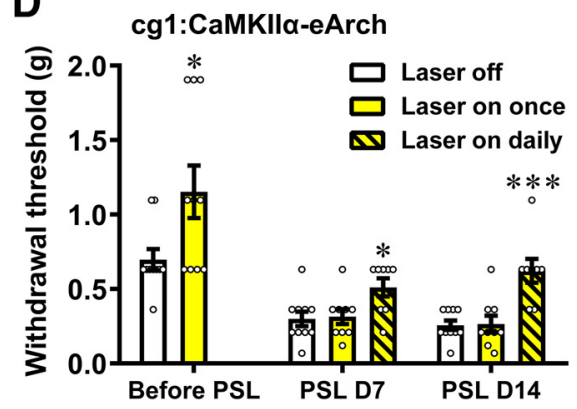

C
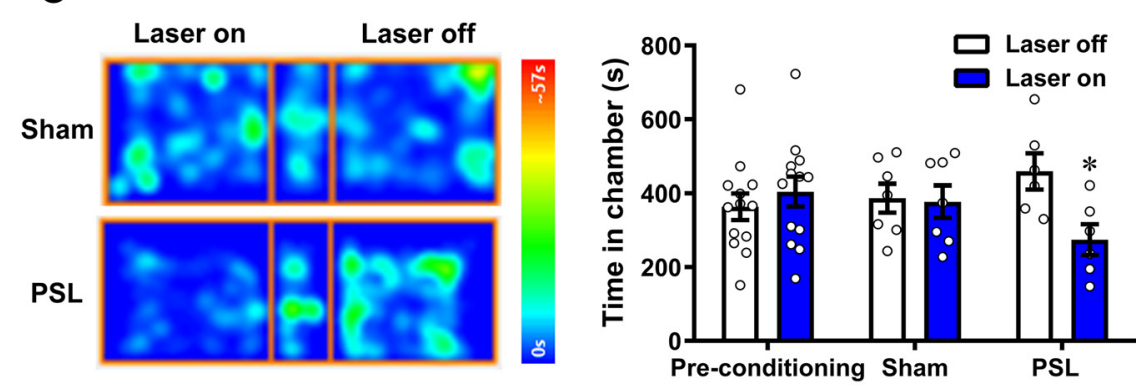

E

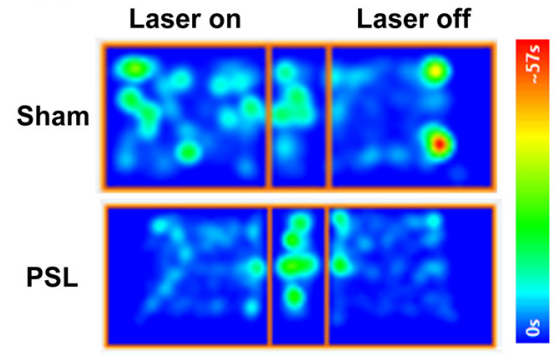

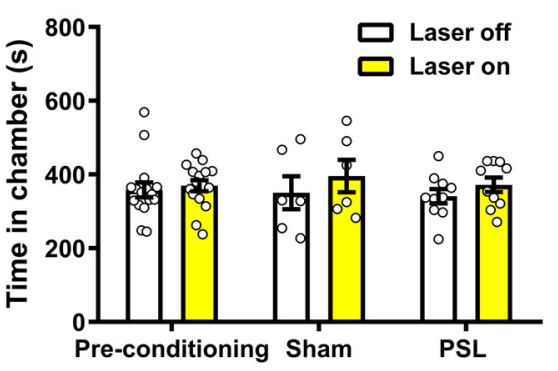

Figure 7. $\mathrm{MCC} \mathrm{Cg} 1^{\text {Glu }}$ play a facilitating role in pain modulation and neuropathic pain. $A$, Schematic drawing of the sites for virus injection and optical stimulation (left) and the expression of ChR2-eYFP in MCC Cg1 (right). Scale bar, $500 \mu \mathrm{m}$. B, Optical activation of unilateral $\mathrm{MCC}\left(\mathrm{Cg}{ }^{\text {Glu }}\right.$ reduced PWT in intact mice and deteriorated allodynia contralaterally in neuropathic mice. Before PSL, $n=10 /$ group, two-tailed unpaired $t$ test, laser on once versus laser off, $p<0.001, t=7.574$, df $=18$. After PSL, laser off $n=10$, laser on once and laser on daily $n=9 /$ group, one-way ANOVA with Tukey's post hoc; $\mathrm{D} 7: F_{(2,25)}=4.478, p=0.0218$, laser on daily versus laser off, $p=0.0235 ; \mathrm{D} 14: F_{(2,25)}=9.294, p=0.001$, laser on daily versus laser off, $p<0.001$. $C$, 0 ptical activation of unilateral MCC Cg1 ${ }^{\text {Glu }}$ induced place aversiveness in neuropathic mice. Sham $n=7, \operatorname{PSL} n=6$, two-way ANOVA with repeated measures and Bonferroni's post-test $\left(F_{(2,46)}=3.352, p=0.0438\right)$. PSL group, test versus preconditioning, $p=0.0407$. D, Optical inhibition of unilateral $\mathrm{MCC} \mathrm{Cg}{ }^{6}{ }^{6}$ ele evated PWT in intact mice and alleviated allodynia contralaterally in neuropathic mice. Before PSL, $n=10$ /group, two-tailed unpaired $t$ test, laser on versus laser off, $p=0.0277, t=2.395, \mathrm{df}=18$. After PSL, laser off $n=10$, laser on once $n=9$, laser on daily $n=8$, one-way ANOVA with Tukey's post hoc. D7: $F_{(2,24)}=4.759, p=0.0182$, laser on daily versus laser off, $p=0.0241 ; \mathrm{D} 14: F_{(2,24)}=13.08, p<0.001$, laser on daily versus laser off, $p<0.001 . E, 0$ ptical inhibition of unilateral $M C C$ $\mathrm{Cg}{ }^{\text {Glu }}$ did not induce place preference in neuropathic mice. Sham $n=6, \operatorname{PSL} n=10$, two-way ANOVA with repeated measures and Bonferroni's post-test $\left(F_{(2,58)}=0.2498, p=0.7798\right) .{ }^{*} p<$ $0.05,{ }^{* * *} p<0.001$.

MCC Cg1 ${ }^{\text {Glu }}$ play a facilitating role in pain modulation and neuropathic pain

These data demonstrate that MCC $\mathrm{Cg} 2{ }^{\text {Glu }}$ participate in pain modulation. Recently, however, Tan et al. (2017) reported that acutely silencing MCC glutamatergic neurons did not affect basal mechanical sensitivity and neuropathic allodynia. Since MCC Cg1 has distinct connections from Cg2 (Fillinger et al., 2017, 2018) and $\mathrm{Cg} 1{ }^{\text {Glu }}$ changed their activity in a different way in response to noxious stimulation as well as in neuropathy (Fig. $1 C, D)$, we further examined the role of $\mathrm{Cg} 1$ Glu in pain modulation and neuropathic pain by optogenetically manipulating their activity after infusing ChR2 or Arch virus targeting MCC Cg1 Glu (Fig. 7A). We found that a brief optical activation of MCC Cg1 ${ }^{\text {Glu }}$ reduced the PWT to mechanical stimulation in the contralateral hindpaw of intact mice (Fig. $7 B$; unpaired $t$ test, $p<0.001$ ), whereas in neuropathic mice, daily photoactivation throughout postoperative days aggravated mechanical allodynia contralaterally (Fig. $7 B$; one-way ANOVA with Tukey's post hoc; D7: $F_{(2,25)}=4.478, p=0.0218$, laser on daily vs laser off, $p=0.0235$; D14: $F_{(2,25)}=9.294, p=0.001$, laser on daily vs laser off, $p<$ $0.001)$. In the CPP test, neuropathic mice spent less time in the blue light-paired chamber, suggesting that activation of MCC $\mathrm{Cg} 1{ }^{\mathrm{Glu}}$ worsened aversiveness associated with ongoing pain (Fig. $7 C$; two-way ANOVA with repeated measures and Bonferroni's post-test: $F_{(2,46)}=3.352, p=0.0438$, PSL group, test vs preconditioning, $p=0.0438)$. On the other side, optical inhibition of MCC Cg1 1 elu evated PWT contralaterally under both physiological (Fig. 7D; unpaired $t$ test, $p=0.0277$ ) and neuropathic conditions (Fig. 7D; one-way ANOVA with Tukey's post hoc; D7: $F_{(2,24)}=4.759, p=0.0182$, laser on daily vs laser off, $p=0.0241$; D14: $F_{(2,24)}=13.08, p<0.001$, laser on daily vs laser off, $p<$ 0.001 ), but did not induce place preference (Fig. $7 E$; two-way 
ANOVA with repeated measures and Bonferroni's post-test: $F_{(2,58)}=0.2498$, $p=0.7798)$. These results suggest that opposite to $\mathrm{Cg} 2{ }^{\mathrm{Glu}}$, MCC $\mathrm{Cg} 1^{\mathrm{Glu}}$ play a facilitating role in pain modulation and neuropathic pain.

\section{Discussion}

Accumulating evidence suggest that both enhanced facilitation and impaired inhibition of pain by some cortical and subcortical structures play roles in NeuP (Chen et al., 2018; Patel et al., 2018). The identification of brain structures that participate in pain modulation and their contribution to NeuP will be of great significance to improve the unsatisfying status of current treatments. Although human brain imaging studies have shown that MCC is consistently and markedly activated by pain (Vogt, 2016a; Kragel et al., 2018), a recent study reported that acutely silencing MCC glutamatergic neurons is not sufficient to affect acute nociception in intact mice, but inhibits capsaicin-evoked hyperalgesia (Tan et al., 2017). It appears that MCC might not be involved in the endogenous pain modulatory circuitry, but contribute to other pain-related responses and pathological pain. However, these studies did not differentiate either types of neurons or subdivisions in the MCC. Here, by selectively manipulating neuronal activity, we found that $\mathrm{Cg} 2{ }^{\mathrm{Glu}}$ and $\mathrm{Cg} 1{ }^{\mathrm{Glu}}$ are both involved in intrinsic pain modulation, but function oppositely. To be specific, $\mathrm{Cg} 2{ }^{\text {Glu }}$ tonically inhibit, but $\mathrm{Cg} 1{ }^{\mathrm{Glu}}$ facilitate, nociception under physiological condition. Although both decrease their activity upon noxious stimulation, their effects on nociception are distinct. We speculate the reason for the failure of acutely silencing MCC glutamatergic neurons to influence mechanical nociception may be that neurons in the two subdivisions were simultaneously manipulated, which leads to the net effect insignificant.

We found that $\mathrm{Cg} 2{ }^{\text {Glu }}$ reduced their firing activity in response to noxious mechanical stimulation and formalin. Given the inhibitory effect on nociception is tonic, the reduced activity should be able to weaken the inhibitory strength, thus facilitate nociception, which would enhance the awareness of tissue damage. Consistently, the inhibitory modulation by activating MCC $\mathrm{Cg}^{\mathrm{Glu}}$ was effective on mechanical nociception and formalin-induced inflammatory pain. These results together support MCC $\mathrm{Cg} 2{ }^{\mathrm{Glu}}$ constitute a component of the endogenous pain modulatory system. Moreover, we found that modulating MCC $\mathrm{Cg} 2{ }^{\text {Glu }}$ activity was only effective on the contralateral inflammatory pain and had no effect on thermal pain. Although sensory inputs are conventionally thought to be transmitted to the contralateral brain, the neuronal circuitry for the transmission and modulation of different types of nociception is currently still elusive (Carr and Zachariou, 2014; De Felice and Ossipov, 2016). Our study identified the selective modulation of MCC Cg2 ${ }^{\text {Glu }}$ on bilateral mechanical nociception and contralateral inflammatory pain, providing evidence for the existence of pain modality-dependent signal transmission and modulation circuitry. Therefore, we propose that, by selectively manipulating certain groups of neurons in certain brain regions, more researches on the pain modulatory system would promote precise pain management in the future medical practice.

After peripheral nerve injury, MCC $\mathrm{Cg} 2{ }^{\text {Glu }}$ became hypoactive. It is conceivable that this change would diminish the strength of pain inhibition and thereby contribute to NeuP. This idea is supported by our findings that selective activation of these neurons alleviated neuropathic allodynia and spontaneous pain. Nonetheless, the pain inhibitory function seems not completely absent after nerve injury because further silencing these neurons deteriorated neuropathic pain. On the contrary, $\mathrm{Cg} 1{ }^{\mathrm{Glu}}$ were hyperactive after nerve injury. Optical manipulation of their activity revealed that this change also contributes to NeuP. These results together demonstrate that both $\mathrm{Cg} 2^{\mathrm{Glu}}$ and $\mathrm{Cg} 1{ }^{\mathrm{Glu}}$ activities shift toward facilitating hypersensitivity after nerve injury. Therefore, by dissecting the roles of $\mathrm{MCC} \mathrm{Cg} 2{ }^{\mathrm{Glu}}$ and $\mathrm{Cg} 1{ }^{\text {Glu }}$ in pain modulation and their dysfunction after nerve injury, we provide new evidence for the concept that NeuP is associated with impaired pain inhibition and enhanced pain facilitation (Ossipov et al., 2010; Apkarian et al., 2011; Kwon et al., 2014). In this regard, the present study supports that enhancement of MCC Cg2 ${ }^{\text {Glu }}$ activity may be used as a therapeutic strategy for NeuP.

In the present study, we identified that the MCC $\mathrm{Cg}_{2}{ }^{\mathrm{Glu}}$ to $\mathrm{ZI}^{\mathrm{GABA}}$ circuit mediates at least in part the analgesic effect of MCC Cg2 ${ }^{\text {Glu }}$, because photoactivation of the terminals in the ZI activated $\mathrm{ZI}{ }^{\mathrm{GABA}}$ and evoked glutamate-dependent analgesic effect. ZI is abundant of GABAergic neurons that have extensive connections with multiple brain regions (Romanowski et al., 1985; Mitrofanis, 2005) and tightly regulate the activity of sensory thalamus (Park et al., 2014). Several lines of evidence have indicated the involvement of $\mathrm{ZI}^{\mathrm{GABA}}$ in NeuP (Lucas et al., 2011; Moon et al., 2016; Moon and Park, 2017). Here we also found that direct activation of $\mathrm{ZI}{ }^{\mathrm{GABA}}$ inhibited pain perception and neuropathic pain. Therefore, hypoactive $\mathrm{MCC} \mathrm{Cg} 2{ }^{\text {Glu }}$ after peripheral nerve injury would weaken the excitatory innervation of $\mathrm{ZI}^{\mathrm{GABA}}$, whereas activation of the $\mathrm{MCC} \mathrm{Cg} 2{ }^{\mathrm{Glu}}$ to $\mathrm{ZI}^{\mathrm{GABA}}$ circuit 
would alleviate neuropathic pain by restoring the analgesic function. We found that MCC $\mathrm{Cg} 2^{\text {Glu }}$ also send projections to the DLPAG. Although the PAG holds the key position in descending pain modulation, its different subregions show different modes of action and are involved in various pain-related functions (Mendes-Gomes and Nunes-de-Souza, 2009; Lei et al., 2014; Sims-Williams et al., 2017). Because photoactivation of axon terminal of MCC Cg2 ${ }^{\text {Glu }}$ in the DLPAG did not affect mechanical sensitivity under either physiological or neuropathic condition, we conclude that the DLPAG plays a minor, if any, role in the analgesic function of MCC Cg2 ${ }^{\text {Glu }}$, but should participate in mediating other functions. Intriguingly, we found that MCC Cg1 ${ }^{\text {Glu }}$ also send axonal projections to $\mathrm{ZI}^{\mathrm{GABA}}$, but this circuit seems not prominently be involved in pain modulation, probably because of the weaker projections to different subdivisions of ZI.

It is worth noting that, although acute activation of the circuit of MCC Cg2 ${ }^{\text {Glu }}$ to $\mathrm{ZI}{ }^{\mathrm{GABA}}$ was able to relieve neuropathic ongoing pain, an accumulative effect resulted from daily activation of either the soma or the ZI terminals was required to alleviate mechanical allodynia. Tan et al. (2017) also found that acute manipulation of MCC glutamatergic neurons does not influence mechanical sensitivity in neuropathic mice. These findings indicate that the compromised analgesic function of $\mathrm{Cg} 2$ Glu after nerve injury was restored only by persistent, but not acute activation of these neurons. It is well documented that glutamatergic synapses display high plasticity both structurally and functionally under a variety of conditions including pathological pain, in which neuronal activity plays an important role (Luo et al., 2014; Reiner and Levitz, 2018). This indicates that the activity of presynaptic neurons per se can influence the synaptic connectivity with postsynaptic neurons. In our case, hypoactive MCC Cg2 Glu should be able to weaken their excitatory strength, which can further lead to changes in the synapses with $\mathrm{ZI}^{\mathrm{GABA}}$. It is very likely that persistent activation of the circuit alleviated neuropathic allodynia by compensating to some extent the weakened synaptic connectivity of MCC Cg2 ${ }^{\text {Glu }}$ with $\mathrm{ZI}^{\mathrm{GABA}}$.

In conclusion, as summarized in Figure 8, the present study identified for the first time that MCC Cg2 ${ }^{\text {Glu }}$ constitute a component of intrinsic pain inhibitory circuitry. MCC Cg2 Glu become hypoactive after peripheral nerve injury and thus underpin NeuP. Our results support that selective and persistent activation of MCC $\mathrm{Cg} 2{ }^{\mathrm{Glu}}$ and the related analgesic circuit may be a therapeutic strategy of NeuP.

\section{References}

Apkarian AV, Hashmi JA, Baliki MN (2011) Pain and the brain: specificity and plasticity of the brain in clinical chronic pain. Pain 152:S49-S64.

Blankstein U, Chen J, Diamant NE, Davis KD (2010) Altered brain structure in irritable bowel syndrome: potential contributions of preexisting and disease-driven factors. Gastroenterology 138:1783-1789.

Bliss TV, Collingridge GL, Kaang BK, Zhuo M (2016) Synaptic plasticity in the anterior cingulate cortex in acute and chronic pain. Nat Rev Neurosci 17:485-496.

Bonin RP, Bories C, De Koninck Y (2014) A simplified up-down method (SUDO) for measuring mechanical nociception in rodents using von frey filaments. Mol Pain 10:26.

Carr FB, Zachariou V (2014) Nociception and pain: lessons from optogenetics. Front Behav Neurosci 8:69.

Chen T, Taniguchi W, Chen QY, Tozaki-Saitoh H, Song Q, Liu RH, Koga K, Matsuda T, Kaito-Sugimura Y, Wang J, Li ZH, Lu YC, Inoue K, Tsuda M, Li YQ, Nakatsuka T, Zhuo M (2018) Top-down descending facilitation of spinal sensory excitatory transmission from the anterior cingulate cortex. Nat Commun 9:1886.

Chou XL, Wang X, Zhang ZG, Shen L, Zingg B, Huang J, Zhong W, Mesik L, Zhang LI, Tao HW (2018) Inhibitory gain modulation of defense behaviors by zona incerta. Nat Commun 9:1151.
Csicsvari J, Hirase H, Czurkó A, Mamiya A, Buzsáki G (1999) Oscillatory coupling of hippocampal pyramidal cells and interneurons in the behaving rat. J Neurosci 19:274-287.

De Felice M, Ossipov MH (2016) Cortical and subcortical modulation of pain. Pain Manag 6:111-120.

Erpelding N, Simons L, Lebel A, Serrano P, Pielech M, Prabhu S, Becerra L, Borsook D (2016) Rapid treatment-induced brain changes in pediatric CRPS. Brain Struct Funct 221:1095-1111.

Fallon N, Chiu Y, Nurmikko T, Stancak A (2016) Functional connectivity with the default mode network is altered in fibromyalgia patients. Plos One 11:e0159198.

Fillinger C, Yalcin I, Barrot M, Veinante P (2017) Afferents to anterior cingulate areas $24 \mathrm{a}$ and $24 \mathrm{~b}$ and midcingulate areas $24 \mathrm{a}^{\prime}$ and $24 \mathrm{~b}^{\prime}$ in the mouse. Brain Struct Funct 222:1509-1532.

Fillinger C, Yalcin I, Barrot M, Veinante P (2018) Efferents of anterior cingulate areas $24 \mathrm{a}$ and $24 \mathrm{~b}$ and midcingulate areas $24 \mathrm{a}^{\prime}$ and $24 \mathrm{~b}^{\prime}$ in the mouse. Brain Struct Funct 223:1747-1778.

Glass JM, Williams DA, Fernandez-Sanchez ML, Kairys A, Barjola P, Heitzeg MM, Clauw DJ, Schmidt-Wilcke T (2011) Executive function in chronic pain patients and healthy controls: different cortical activation during response inhibition in fibromyalgia. J Pain 12:1219-1229.

Hubbard CS, Khan SA, Keaser ML, Mathur VA, Goyal M, Seminowicz DA (2014) Altered brain structure and function correlate with disease severity and pain catastrophizing in migraine patients. eNeuro 1:ENEURO.0006-14.2014.

King T, Vera-Portocarrero L, Gutierrez T, Vanderah TW, Dussor G, Lai J, Fields HL, Porreca F (2009) Unmasking the tonic-aversive state in neuropathic pain. Nat Neurosci 12:1364-1366.

Kragel PA, Kano M, Van Oudenhove L, Ly HG, Dupont P, Rubio A, DelonMartin C, Bonaz BL, Manuck SB, Gianaros PJ, Ceko M, Reynolds Losin EA, Woo CW, Nichols TE, Wager TD (2018) Generalizable representations of pain, cognitive control, and negative emotion in medial frontal cortex. Nat Neurosci 21:283-289.

Kulkarni B, Bentley DE, Elliott R, Julyan PJ, Boger E, Watson A, Boyle Y, El-Deredy W, Jones AK (2007) Arthritic pain is processed in brain areas concerned with emotions and fear. Arthritis Rheum 56:1345-1354.

Kwon M, Altin M, Duenas H, Alev L (2014) The role of descending inhibitory pathways on chronic pain modulation and clinical implications. Pain Pract 14:656-667.

Lei J, Sun T, Lumb BM, You HJ (2014) Roles of the periaqueductal gray in descending facilitatory and inhibitory controls of intramuscular hypertonic saline induced muscle nociception. Exp Neurol 257:88-94.

Le Van Quyen ML, Bragin A, Staba R, Crépon B, Wilson CL, Engel J Jr (2008) Cell type-specific firing during ripple oscillations in the hippocampal formation of humans. J Neurosci 28:6104-6110.

Lucas JM, Ji Y, Masri R (2011) Motor cortex stimulation reduces hyperalgesia in an animal model of central pain. Pain 152:1398-1407.

Luo C, Kuner T, Kuner R (2014) Synaptic plasticity in pathological pain. Trends Neurosci 37:343-355.

Mendes-Gomes J, Nunes-de-Souza RL (2009) Anxiolytic-like effects produced by bilateral lesion of the periaqueductal gray in mice: influence of concurrent nociceptive stimulation. Behav Brain Res 203:180-187.

Mills EP, Di Pietro F, Alshelh Z, Peck CC, Murray GM, Vickers ER, Henderson LA (2018) Brainstem pain-control circuitry connectivity in chronic neuropathic pain. J Neurosci 38:465-473.

Mitrofanis J (2005) Some certainty for the "zone of uncertainty"? exploring the function of the zona incerta. Neuroscience 130:1-15.

Moon HC, Park YS (2017) Reduced GABAergic neuronal activity in zona incerta causes neuropathic pain in a rat sciatic nerve chronic constriction injury model. J Pain Res 10:1125-1134.

Moon HC, Lee YJ, Cho CB, Park YS (2016) Suppressed GABAergic signaling in the zona incerta causes neuropathic pain in a thoracic hemisection spinal cord injury rat model. Neurosci Lett 632:55-61.

Nicolelis MA, Chapin JK, Lin RC (1992) Somatotopic maps within the zona incerta relay parallel GABAergic somatosensory pathways to the neocortex, superior colliculus, and brain-stem. Brain Res 577:134-141.

Ossipov MH, Dussor GO, Porreca F (2010) Central modulation of pain. J Clin Invest 120:3779-3787.

Park A, Hoffman K, Keller A (2014) Roles of $\mathrm{GABA}_{\mathrm{A}}$ and $\mathrm{GABA}_{\mathrm{B}}$ receptors in regulating thalamic activity by the zona incerta: a computational study. J Neurophysiol 112:2580-2596.

Patel R, Qu C, Xie JY, Porreca F, Dickenson AH (2018) Selective deficiencies 
in descending inhibitory modulation in neuropathic rats: implications for enhancing noradrenergic tone. Pain 159:1887-1899.

Paxinos G, Franklin KBJ (2001) The mouse brain in sterotaxic coordinates. San Diego, CA: Academic Press.

Porreca F, Ossipov MH, Gebhart GF (2002) Chronic pain and medullary descending facilitation. Trends Neurosci 25:319-325.

Reiner A, Levitz J (2018) Glutamatergic signaling in the central nervous system: ionotropic and metabotropic receptors in concert. Neuron 98:1080-1098.

Romanowski CA, Mitchell IJ, Crossman AR (1985) The organization of the efferent projections of the zona incerta. J Anat 143:75-95.

Seltzer Z, Dubner R, Shir Y (1990) A novel behavioral-model of neuropathic pain disorders produced in rats by partial sciatic-nerve injury. Pain 43:205-218.

Sims-Williams H, Matthews JC, Talbot PS, Love-Jones S, Brooks JC, Patel NK, Pickering AE (2017) Deep brain stimulation of the periaqueductal gray releases endogenous opioids in humans. Neuroimage 146:833-842.

Tan LL, Pelzer P, Heinl C, Tang W, Gangadharan V, Flor H, Sprengel R,
Kuner T, Kuner R (2017) A pathway from midcingulate cortex to posterior insula gates nociceptive hypersensitivity. Nat Neurosci 20:1591-1601.

Vogt BA (2005) Pain and emotion interactions in subregions of the cingulate gyrus. Nat Rev Neurosci 6:533-544.

Vogt BA (2016a) Cytoarchitecture and neurocytology of rabbit cingulate cortex. Brain Struct Funct 221:3571-3589.

Vogt BA (2016b) Midcingulate cortex: structure, connections, homologies, functions and diseases. J Chem Neuroanat 74:28-46.

Wang Y, Xu C, Xu Z, Ji C, Liang J, Wang Y, Chen B, Wu X, Gao F, Wang S, Guo Y, Li X, Luo J, Duan SM, Chen Z (2017) Depolarized GABAergic signaling in subicular microcircuits mediates generalized seizure in temporal lobe epilepsy. Neuron 95:92-105.e5.

Yuan J, Cao S, Huang Y, Zhang Y, Xie P, Zhang Y, Fu B, Zhang T, Song G, Yu T, Zhang M (2018) Altered spontaneous brain activity in patients with idiopathic trigeminal neuralgia: a resting-state functional MRI study. Clin J Pain 34:600-609.

Zimmermann M (1983) Ethical guidelines for investigations of experimental pain in conscious animals. Pain 16:109-110. 